\title{
Die Attische Königsliste.
}

\author{
Von F. Jacoby.
}

I.

Die Listen der Chronogriphen.

Die attische Königsliste ist vielfach behandelt worden. Auch haben die ihr gewidmeten Forschungen wenigstens für die Frage nach der Entstehung ihres ersten Teiles, der Könige von Kekrops bis Thymoites, manche als sicher zu bezeichnende Resultate geliefert. Allerdings lag die Sache hier insofern einfach, als die Entwicklung, d. h. die allmähliche Erweiterung dieses Teiles der Liste sich fast vor unseren Augen vollzog, wenn wir auch die einzelnen Stadien dieser Entwicklung und die Namen der Schriftsteller, durch die die Etappen zu bezeichnen sind, nicht überall mit voller Sicherheit nennen können. Über den chronologischen und historischen Wert dieses Produktes aus der Zeit um die Wende des 5. und 4. Jahrhunderts besteht kein Zweifel; nur über die Bedeutung der wenigen alten Elemente, die es enthält, wie etwa über das Verhältnis von Kekrops zu Erechtheus, ist eine Erörterung möglich, allerdings nur eine solche ethnographischen Charakters.

Sehr viel anders aber steht es mit dem zweiten Teile der Liste, mit

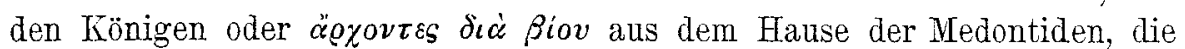
in unserer Überlieferung von den sogenannten Erechtheiden durch das Zwischenglied der beiden Melanthiden getrennt werden, und mit den

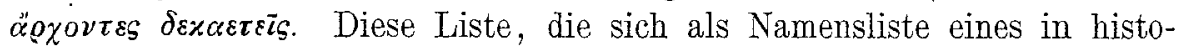
rischer Zeit in Athen nachweisbaren Adelsgeschlechtes giebt, zeigt ein wesentlich anderes Gesicht, als der aus wenigen mythischen und vielen Füllfiguren bestehende erste Teil. Die Fragen, die sich hier erheben, sind daher viel gewichtiger. Was ist historisch an dieser Gliederung der

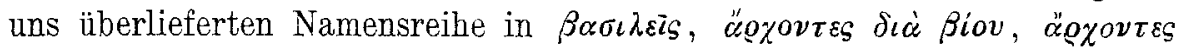

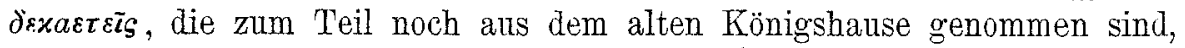
zum Teil aus der Gesamtheit der Adelsgeschlechter? Und was ergiebt sich darans für die Verfassungsgeschichte Athens? Wie weit geht der chronologische Wert der etwa als historisch erkannten Überlieferung? 
Der Versuch, diese Fragen zu beantworten, hat zwei Stadien zu durchlaufen, die man kurz als das chronographische und das historische bezeichnen kann. Es gilt in erster Linie, die von den alten Chronologen aufgestellte Liste oder auch Listen wieder zu gewinnen, indem wir sie aus der zum Teil späten und schlechten Überlieferung soweit wie möglich in ihren Einzelheiten rekonstruieren; dann aber die Entstehung dieser chronologischen Systeme und die sie beherrschenden Prinzipien zu verstehen. Erst die sorgfältige, die Überlieferung klarlegende Durchführung. dieser Aufgabe ermöglicht, indem sie zeigt, was die antiken Gelehrten von den Königen Athens zu wissen glaubten, das Urteil über den historischen Wert der ganzen Liste oder ihrer einzelnen Teile und über ihre Branchbarkeit für die Erkenntnis der Verfassungsgeschichte Athens. Denn ein solches chronographisches System zeigt, wenn es in seiner Reinheit wiederhergestellt ist, meist sehr dentlich, wo die Überlieferung aufhört und die Konstruktion anfüngt. Diese Erkenntnis leitet dann über zu der Frage, was in dem so begrenzten Teile historisch ist, ohne doch chronologischen Wert zu haben, und was mythisch oder reine Fiktion ist; woher die mythischen Elemente stammen, was sie etwa bedenten und wie man. dazu kam, sie in die Köniosfasten aufzunehmen, schliesslich welchem Zweck die rein fiktiven Elemente ihre Entstehung verdanken. Aber alle diese Fragen darf man erst angreifen, wenn die chronographische Tradition vorgelegt ist. Während dieses Prozesses sind alle historischen Betrachtungen und Exkurse vom Übel, weil sie die Sicherheit des Resnltates - die Rekonstruktion der antiken Ansicht - gefährden, indem sie uns vorgefasste Meinungen über die Entwicklung der Königsmacht in den antiken Systemen vorspiegeln.

Für die chronologische Untersuchung der attischen Königsliste sind wir in einer verhältnismässig glïcklichen Lage, weil die uns vorliegende Überlieferung keine einheitliche ist. Wir besitzen die Liste vielmehr in zwei Rezensionen, aus deren konsequenter Vergleichung sich uns wertvolle Resnltate für ihren chronographischen Wert ergeben werden; es sind erstens die Rezension der im Jahre $263 / 2$ a. Chr. ${ }^{1}$ ) verfassten parischen Marmorchronik , ${ }^{2}$ ) deren Rückgrat die einer Atthis entlehnte Königsliste

1) Wenigstens will der Verfasser der Chronik die Ereignisse aufzeichnen åo $\dot{\alpha}^{\prime}-$

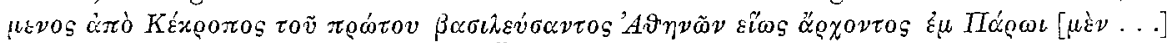

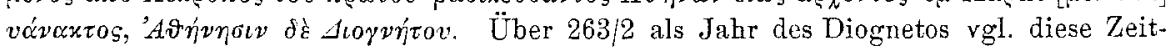
schrift II S. $163-165$.

2) Brauchbare Ausgabe nur von Boвскн, CIG. II 2374 (S. 293 if.). Die Spezialausgabe von FisCH, Chronicon Parium, Tübingen 1884 ist in ihren chronographisehen Darbietungen noch wertloser als in dem sachlichen Kommentar. Nach dem Funde des neuen grossen Stuckes der Inschrift (Athen. Mittheil. XXII 1897 S. $183 \mathrm{ff}$.) sind Neupublikationen in Vorbereitung von Hrtuer v. Gartringles im Inselcorpus, von J. A. R. Munro (s. seine vorläufigen Mitteilungen in Classical Review XV 1901 S. 149-154, $355-361$, von F. JAcoby. 
bildet; zweitens die Liste Kastors aus dem 1. Jahrlundert a. Chr., ${ }^{1}$ ) die uns von den christlichen Chronographen erhalten ist und deren Entstehung, wie sich unten ergeben wird, höchstwahrscheinlich ebenfalls bis in das 3. vorchristliche Jahrhundert hinaufreicht. Sie wird, weil sie vollständig vorliegt, zuerst zu besprechen sein.

\section{Die attische Königsliste Kastors.}

In seinen tief einschneidenden Untersuchungen über die grossen chronographischen Systeme des späten Altertums, die den bescheidenén Titel Die Königslisten des Eratosthenes und Kastor tragen, ${ }^{2}$ ) hat Edvard Schwartz auch die attische Liste Kastors als die wichtigste von allen eingehend und scharfsinnig behandelt. Durch Nebeneinanderstellung und Vergleichung der verschiedenen Zweige der Überlieferung, in denen uns Eusebios' Liste erhalten ist, hat er sich bemüht, die Grundform, die nach Eusebs eigener Angabe $^{3}$ ) dem grossen chronographischen Werke Kastors entnommen ist, auch in ihren Einzelheiten wiederherzustellen. Besonders wichtig ist, dass er die sogenannten Excerpta Barbari ${ }^{4}$ ) als Zweig dieser Eusebischen Überlieferung, dessen Bedentung er allerdings zuweilen überschätzt, erwiesen hat, ohne dabei ihre gelegentliche starke Interpolation aus dem System des Africanus zu verkennen. Durch diesen Nachweis ist den zahlreichen Vermutungen, die man auf Einzelabweichungen der Excerpta gegründet hat, der Boden entzogen. Bei der geringen Beachtung, die Schwartz' Arbeit gefunden hat - man kann das ihr gegenüber beobachtete Verfahren nur mit Totschweigen bezeichnen ${ }^{5}$ ) - ist es zu einer Nachprüfung seiner Resultate noch kaum in einem Punkte gekommen. Und doch erscheint mir eine solche Nachprifung, wie ich sie an anderer Stelle

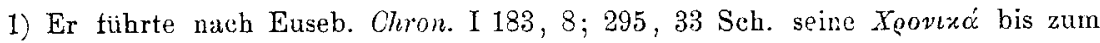
Jahre des Theophemos herab, d. h. $61 / 0$ a. Chr. (vgl. Kincraner, Prosop. Atticu no. 7092).

2) Aus den Abhandlungen der Konigl. Gesellschaft der Wissenschuften in Gottingen, Band XL, 1894.

3) Euseb. Chron. I 181, $30 \mathrm{ff}$ : Kastoris de Regno Atheniensium. Kanon a. Abr. 880. 887.

4) Eusebii Chronic. I appendix VI p. $216 \mathrm{f}$. Schorne.

5) Soviel ich weiss, ist nur eine einzige Rezension der Königslisten erschienen; es ist die kurze Anzeige TópfFens im Litterar. Centralblatt 1895 (- Beiträge zur griechischen Altertumswissenschaft von JoHanNes TöpfFER 1897, S. $271 \mathrm{ff}$.), die durch einen Aufsatz im Hermes 1896 - Beitrage S. $275 \mathrm{ff}$.) Die Liste der athenischen Konige ergänzt wird. Die Rezension beschäftigt sich eigentlich nur mit der Lykurgfrage, ohne weiter auf die chronologischen Untersucbungen einzugehen, die auch in dem Aufsatz ubber die attische Liste nur kurz und, wie mir scheint, nicht allzu glücklich beruhrt sind. - In dem kürzlich erschienenen Aufsatze von H. Peter, 'Die Epochen in Varros Werk De Gente Populi Romani. Rh. Mus. LVII, 1902, S. $231 \mathrm{ff.}$, der sich viel mit Kastors Chronologie beschäftigt, findet man Schwar'z' Nanen überbaupt nicht erwähnt. [Vgl. aber andererseits Montzkas obige Abhandlung. Red.] 
fïr andere Partieen zu geben versucht habe, $\left.{ }^{1}\right)$ auch für die attische Liste keineswegs überflüssig. Denn auch hier ist Scuwartz, wie mir scheint, durch geistvolle, aber gewagte Kombinationen und durch rasche zu wenig abgewogene Entscheidungen über die Diskrepanzen der Überlieferung, zu einer Gestaltung der Liste Kastors gekommen, die mir in verschiedener Hinsicht nicht einwandfrei zu sein scheint. Freilich wird durch eine solche revidierende Untersuchung SchwarTz Hauptresultat, das ich in der Beurteilung des Eusebischen Kanons sehe, nicht umgestossen, sondern nur in einzelnen Punkten modifiziert. Geschalet hat auch dieser Untersuchung meines Erachtens besonders der Glaube, dass wichtige historische Daten aus dieser Liste sich gewinnen liessen, ein Glaube, der die Rekonstruktion der Liste unwillkürlich beeinflusst hat. Ich habe schon oben bemerkt, wie schädlich dieses Hereinziehen moderner Hypothesen über die historische Entwicklung des attischen Königtums den rein chronographischen Ergebnissen, die wir znerst gewinnen müssen, werden kann. Ganz gleichgiltig, ob die Hypothesen glaubwürdig sind oder nicht; das Resultat wird immer dem Einwande begegnen, nicht das historische Faktum ergäbe sich aus dem chronographischen Thatbestand, sondern die chronographische Überlieferung sei zurechtgerückt um der historischen Hypothese willen. Der Einwand wird um so sicherer erhóben werden, je mehr gerade am entscheidenden Punkte die Überlieferung verschiedene Wege zur Rekonstruktion der ursprünglichen Liste zu erlauben scheint.

Mit vollem Recht hat man, seit überhaupt das Verständnis für das Verfahren der antiken Chronographen eröffnet ist, für jede Liste oder für jedes chronographische System ${ }^{2}$ ) die Notwendigkeit eines festen Ausgangspunktes betont, des Epochenjahres, das natürlich nicht das erste Jahr der ganzen Liste ist, sondern das erste historisch sichere, von dem ans die Fasten nach rïckwärts hergestellt sind. Bei der attischen Liste sind wir keinen Augenblick im Zweifel, wo dieser feste Punkt zu suchen ist. Wir

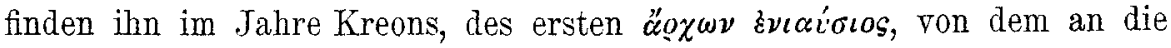
Aufzeichnung der eponymen Beamten in ununterbrochener Folge den späteren Historikern vorlag und die Möglichkeit kurzer sicherer Datierung

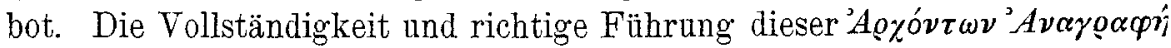
ist mit Grund nicht zu bezweifeln. ${ }^{3}$ ) Ihr Anfangsjahr festzustellen, muss

1) Philol. Untersuch. (herausg. von Krgssurng und v. Wid amowitz-Mólendorer) XVI (1902) S. 80 ff. für die spartanischen Fasten; ebenda S. 91 ff. für die korinthischen; ebenda S 139, 4 für das Daturn der Einsetzung des Ephorates.

2) "Jede nicht rein historische Liste ist bei den griechischen Chronographen ein System", sagt Schwartz, Konigslisten S. 77 mit Recht.

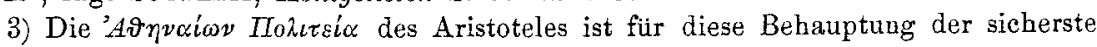
Beweis. Darauf ist oft hingewiesen. Aristoteles setzt eine Archontenliste in den Händen seiner Leser voraus. In dieser aber giebt es keine Differenzen. Der einfache Hinweis auf sie geniigt, um chronologische Fragen zu entscheiden (c. XVII 2). Die Anarchiejahre werden ebenso sorgfältig bezeichnet, wię längere Usurpationen (so die 
also unsere erste Aufgabe sein. Dann erst ist die sichere Grundlage für die Restitution des ïlteren Teiles der Liste gewonnen. Wenn SoHwartz statt dessen von dem vorkreontischen Teile ausgeht, diesen in den verschiedenen Zweigen der Überlieferung vorlegt und so die zu Grunde liegenden Daten Kastors zu gewinnen sucht, so kann dieses Fortschreiten vom unsicheren zum sicheren, vom festzustellenden zum feststehenden nicht ohne Gewaltsamkeiten abgehen. So geht er denn auch, als er endlich an Kreons Jahr kommt, mit einem kïhnen Satze über die gerade hier liegenden Schwierigkeiten, die er sich allerdings zum Teil selbst geschaffen hat, fort. „Denn $682 / 1=01.24,3$ als erstes Jahr der Archontenliste ist nicht zn bezweifeln, da Dionys $[A R$. I 71,5] Charops 1. Jahr $=01.7,17521$

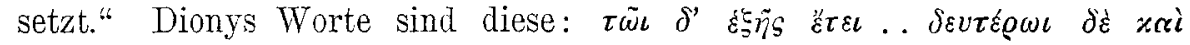

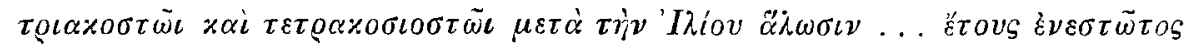

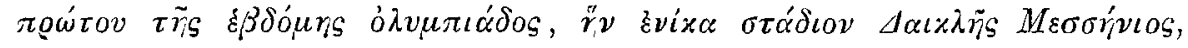

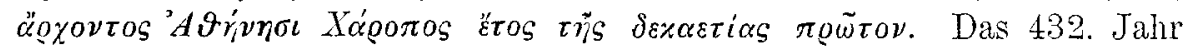
nach Troias Fall $(1184 / 3)$ ist $752 / 1=01.7,1.70$ Jahre für die $7 \not ̈ o \chi o v \tau \varepsilon s$ $\delta \varepsilon x \alpha \varepsilon \tau \varepsilon \check{c}_{\varsigma}$ ergeben $682 / 1=01.24,3$ als Jahr des Kreon. Das scheint plan. Niemand könnte widersprechen, ${ }^{1}$ ) wenn wir nicht noch einige andere Zeugnisse hätten. Zwar dass verschiedene Zeitangaben des Pausanias mit diesem Resultat nicht stimmen, ${ }^{2}$ ) lhat ScHWdR'\% mit Recht als

des Damasias) oder das Archontat der zehn Männer (c. XIII 1. 2). Von dem irregulären

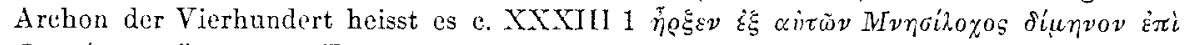

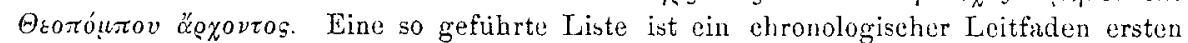
Ranges. Es ist unverstïndlich, wie selbst Busolt, $G r . G$ Ir ${ }^{2} 258,3$ daran glauben

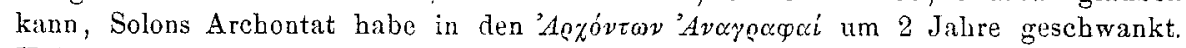
Vgl. daruber Philol. Unters. XVI S. $165 \mathrm{ff}$. Die Modernen schaffen sich hier mutwillig Schwierigkeiten. - Dass die Archontenliste auch für das 7. Jabrb. ebenso sicher vorlag, zeigt wieder die einfache Datierung der $\vartheta c \sigma u o \vartheta \varepsilon \sigma i c$ Drakons $\dot{\varepsilon} \pi$ ' 'A

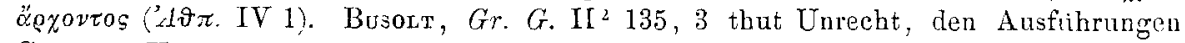
Gerzens, Kastors attische Königs- und Avchontenliste in den Histor. und philolog. Aufsatzen $\dot{f}$. E. Curtius 1884 S. $11 \mathrm{ff}$. zu folgen. Fur Kreon hat es nur ein Datum gegeben; uicht drei oder vier. Eine Bemerkung wie die v. Schoegrens vor seiner : ̈usserst

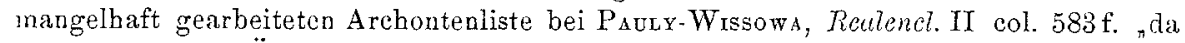
eine einheitliche Überlieferung uber die Archonten des 7. Jahrb. wohl kaum existiert bat, wäre der Versuch, die aus verschiedenen Quellen erhaltenen Daten in Übereinstimmung zu bringen, vergeblich" halte ich fur sehr bedenklich.

1) Obwohl es Sorwartz selost thut bei der Besprechung von Africanus' attiseher Liste. Vgl. S. 418,1 zu Ende.

2) Pausanias setzt IV 5, 10 Aisimides' 5. Jahr = Ol. 9, 2 743/2; IV 13, 7 Hippomenes' 4. Jahr $=01.14,1$ 724/3. Das wurde für Kreon 687/6 ergeben. Ein derartiges

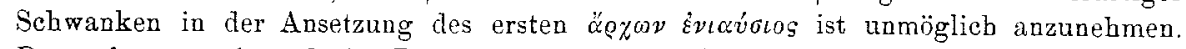
Pausanias muss irgend eine Dummbeit gemacht haben. An Zahlenkorruptel ist jedenfalls nicht zu denken. Nach der Liste von Schwartz ist Aisimides' כ. Jahr = Ol. 10, 3 738/5, Hippomenes' 4. Jahr $=01.15,2$ 719/18; nach'der meinigen (s. S. 420) sind die entsprechenden Jahre vielmehr Ol. 10, $2739 / 8$ und Ol. 15, 1 720/19, d. h. Pausanias irrt genau um 1 Olympiade; und dieser Fuhler geht durch diese ganze Partie seines Werkes durch; denn IV 15, 1 setzt er Tlesias auf Ol. 23,4 685/4 und fugt hinzu: 
gleichgiltig behandelt. ${ }^{1}$ ) Auch Eusebios kann man nicht so gegen ihn ins Feld führen, wie Topfrer das thut, der sich die Sache doch etwas zu

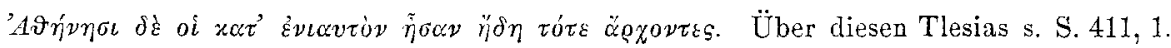
Auch bier scheint der Irrtum gerade 1 Olympiade zu betragen. Wie er entstanden ist, lässt sich nur vermuten. Ich glaube aber, dass er sich mit der pointierten Antithese von Schwartz, Königslisten p. 18, 1, dass der Perieget nentweder eine gute Tabelle schlecht oder eine schlechte gut abgeschrieben hat" crledigen lässt. Tóprrers Widerspruch gegen diese Formulierung (Beatrage S. 286, 1) scheint mir jedenfalls unberechtigt. Denn wenn er annimmt, Pausanias' Liste babe in ibrem äIteren 'Teile um 3 Tahre von der des Pariers differiert, so war es eben eine schlechte Liste und Pausanias hat sie gut abgescbrieben. Das ist Schwartz' zweite Möglichkeit. Ich neige persönlich zu Uvaers Ansicht (Die troische Ära des Suidas 1885 S. 71), der den scheinbar um 4 Jahre zu frühen Beginn von Pausanias' Liste durch zweimaliges zählen der Archonten einer Olympiade erklärt. Nach der Olympionikenliste des EratosthenesAfricanus bei Euseb. Chronic. I 197, $12 \mathrm{ff}$. hat der Lakone Chionis dreimal zu Olympia im Stadion gesiegt, nämlich Ol. 29 664, 01. 30 660, 01. 31 656. Dagegen sagt Pausan.

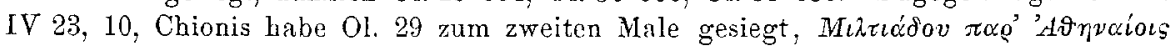

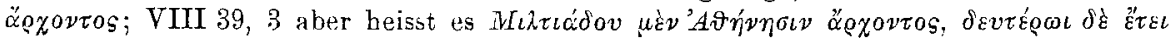

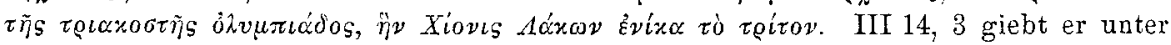
Berufung auf eine Inscbrift ( $\mathrm{vgl}$. auch VI 13, 2) dem Chionis 4 Stadionsiege in Olympia;

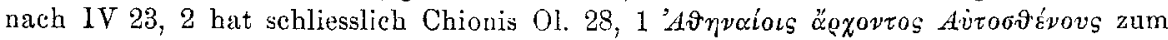
ersten Male gesiegt, der 4 . Sieg wirde also in 01. 31 zu setzen sein. Nun könnte man ja diese Sehwierigkeit dadurch beseitigen, dass man in der Liste des Eusebius den Sieger von Ol. 28 Xóouls Á́xwv als verschricben fur Xiovss ansïhe. Aber dagegen

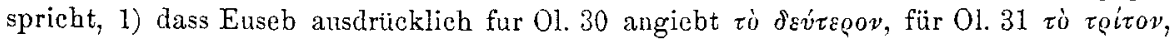

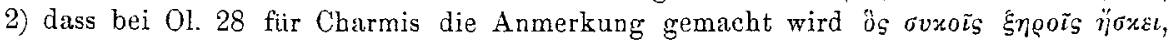

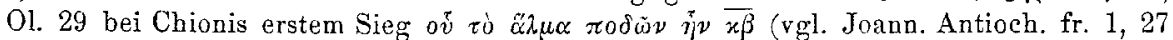
FHG. IV 540). Offenbar hat der Verfasser der Liste, d. i. Eratosthenes, Cbarmis und Chionis fur zwei verschiedene Personen gehalten. Nun könnte freilich immer noch Pausanias Charmis in Chionis verlesen haben. Aber es ist doch seltsam, dass er sowohl für O1. 29, wie für O1. 30 den Archon Miltiades nennt. Das hindert uns, uns bei der Konstatierung, Pausanias habe eben einen anderen Olympionikenkatalog benutzt wie Africanus, zu beruhigen und spricht entsehieden fur Uvgers Ansicht. - Wie dem aber sei; Pausanias kann jedenfalls nicht benutzt werden, Scuwsr's' Ansatz für Kreon zu erschuttern. Doeb s. die nächste Anmerkung. Übrigens ist dem Pausanias ein gauz analoger Irrtum in der Zählung der Pythiaden passiert (X 7, 4), dessen Veranlassung wir noch erkennen. Seine 1. Pythiade liegt 4 Jahre friber als bei unseren sonstigen Zeugen; und der Grund ist eine blosse Flüchtigkeit des Periegeten.

1) In einem Falle könnte freilich Pausanias doch fü den Ansatz Kreon $=682 / 1$ verhängnisvoll werden. IV 15, 1 sełzt er, wie bemerkt (S. 410, 2) Tlesias in Ol. 23, 4 $685 / 4$ und fuggt binzu 'A Irrtum um 1 Olympiade konstatiert ist, so gehört Tlesias vielmebr in Ol. 24, 4 681/0 und wird auch allgemein so angesetzt. Diesen Tlesias aber identifiziert man seit langem mit dem Eponymos von Marmor Parium ep. 33, für dessen Namen jetzt (s. Munro,

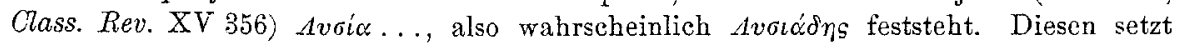
der Chronist 418 Jahre vor seine Epoche, also in Ol. 24, 3 682/1 oder 24, $4681 / 0$. Es ist ja möglich, eine Variante im Namen des Archons von 681,0 anzunehmen. Solche Varianten sind häufiger (einige Beispiele bei B. KeIL, Anonymus Argentinensis S. 30). Jedenfalls aber ist es unwahrscheinlich, wie es seit Lepaulmren die Herausgeber, auch Bовсвн thun, das Marmor aus Pausanias zu korrigieren. Der alte Zeuge aus dem 3. Jahrh. a. Chr. hat jedenfalls eher das richtige bewahrt. Gegen die Korrektur des 
leicht gemacht hat. Wenn Euseb im 1. Buche der Chronik und die Excerpta Barbari Kreon kurzweg Ol. 24 ansetzen, ${ }^{1}$ ) so ist der Schluss, dass damit das erste Jahr gemeint sei, keineswegs zwingend. Dazu sind Nachlässigkeiten in der Angabe der Olympiadenjahre oder Ausfall in der schlechten Überlieferung viel zu häufig. Im Kanon aber ist beim Armenier wie bei Hieronymus das Abrahamsjahr 1333 das letzte des Eryxias, 1334 erstes der évı $\alpha \dot{v} \sigma \iota \iota$. Also Kreon

beim Armenier: a. Abr. $1334=01.24,3=682 / 1$

bei Hieronymus: a. Abr. $1334=01.24,2=683 / 2$

Da nun allein Hieronymus die Olympiaden richtig geglichen hat,$\left.{ }^{2}\right)$ der Armenier aber durch den falschen Ansatz Ol. 1, 1=a. Abr. 1240 (statt 1241) stets um 1 Olympiadenjahr voraus ist, so hat Eusebios Ol. 24, 2 als Jahr Kreons gegeben.

Aber der Kanon ist interpoliert, wie Schwartz das nachgewiesen lat. Wan kann nicht von vornherein leugnen, dass die Interpolation der Regierungszahlen, falls eine solche erfolgt ist, nicht auch eine Verschiebung. von Kreons Jahr verursacht habe, wiewohl sich ein Grund für die Interpolation schwer absehen lässt. Warum sollte der Interpolator den Schlussstein der Liste verrïcken, wenn er seine Zwecke - es liandelt sich bei der attischen Liste eigentlich nur um die durch Herabschiebung von Eusebs troischem Datum 1184;3 auf 1182/1 nötig gewordenen Änderungen durch Änderung der Einzelregierungen sicherer und unauffälliger erreichen konnte und erreicht hat? Auch Interpolation aus Africanus kann hier nicht in Frage kommen, da dessen Ansatz für Kreon der gleiche war wie der des Eusebios.") Auf eine Linie z. B. mit den im Kanon geradezu unglaublich misshandelten spartanischen Fasten kann man die athenischen auch nicht stellen. Denn bei jenen ist der Grund der Interpolation wenigstens bis zu einem gewissen Grade verständlich. Obwohl aber das spartanische Filum völlig verschoben ist, sind so reichliche Spuren des ursprünglichen vorhanden, dass die Beseitigung der Verwüstungen unschwer erfolgt. ${ }^{4}$ ) All das trifft für das athenische Filum nicht zu. Aber

Tlesias aber in Lysiades spricht wieder die Seltenheit des Namens Tlesias. Das einfachste wäre es daher, die Identifikation der beiden Namen aufaugeben und anzunebmen, dass der Chronist ep. 33 von $264 / 3$ gerechnet hat; denn diese Berechnung ist auch dem ersten Teile des Marmors keineswegs fremd (s. S. 414, 1). Dann bekommen wir die Reihe 683/2 Kreon (Marmor), 682/1 Lysiades (Marmor), 681/0 Tlesias (Pausanias). Damit wäre 682/1 für Kreon unmöglich.

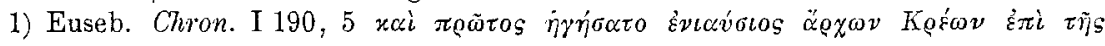

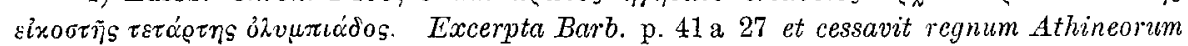
in olympiada vicesima quarta. Series Regum p. 11 Schoеsu: desierunt XXIV. olompiade.

2) Vgl. auch daruber Schwartz, Konigslisten S. 22 ff. und unten S. 419.

3) Über Africanus' Datum für Kreon vgl. S. 418, 1.

4) Über die spartaniseben Fasten bei Eusebios vgl. Schwartz a. a. O. S $60 \mathrm{ff}$. JАсовт, Philol. Unters. XVI S. 80 ff. Den Grund der Interpolation, durch die das 
einerlei; nachdem nun einmal Interpolationen im Kanon nachgewiesen sind, muss der Unschuldige mit dem Schuldigen leiden. Es ist sicherer, kein Datum des Kanons als Ausgangspunkt zu benutzen, solange man es nicht anderweitig sichern kann. Glücklicherweise ist eine solche Sicherung. hier möglich. Denn noch haben wir den wichtigsten Zeugen nicht vorgeführt. Im Marmor Parium heisst es Ep. 32 $\alpha \varphi^{\prime}$ ờ $x \alpha \tau^{\prime} \dot{\varepsilon} \nu \iota \alpha v \tau \dot{o} \nu$

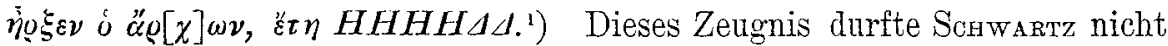
en bagatelle behandeln, indem er es in eine Anmerkung verwies. „Daten des Marmor Parium lassen sich, wenn keine bekannten Eponymen gegeben werden, nicht aufs Jahr berechnen", bemerkt er dazu (p. 18, 1). Das ist freilich richtig. Aber wir haben die Wahl eben nur zwischen zwei Jahren, und das sind hier Ol. 24, 1 684/3 $(264 ; 3+420$ sog. Computus A) und Ol. 24, 2 683/2 (263/2 + 420 sog. Computus B). Ol. 24, 3 $682 / 1$ ist unmöglich, es sei denn man schlage den Verzweiflungsausweg ein, in der Epochenzahl einen Fehler des Steinmetzen zu sehen. Daran aber wird bei dem Charakter der Zahl HHHHAA niemand glauben. Also 684/3 oder $683 / 2$ war Kreon Archon. ${ }^{2}$ ) Erinnern wir uns jetzt, dass

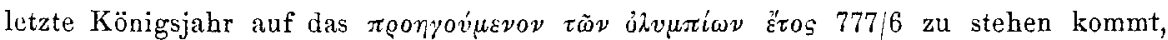
hat auch Schwartz nicht ganz aufgeklïrt. Denn die Einsetzung des Ephorates und damit die Benutzung der Ephorenliste statt der Königsjabre zu chronologischen $Z_{\text {wecken }}$ - was der Interpolator dahin missverstanden bat, dass mit Einsetzung des Ephorates die Königsherrschaft abgeschafft worden sei - fällt in Ol. 6, $3754 / 3$ (s. Philol. Unters. YVI $138 \mathrm{ff}$.); und dieses Datum findet sich mit leichter Verschiebung auf Ol. 5, 4 [5, 1 F. 5, 3 PM. 6, 1 Armen ] noch jetzt im Kanon: in Lacedaemone primus éqoọos, quod magistratus nomen est, constituitur. Fuit autem sub regibus Lacedaemon annis CCCL. Beim Armenier steht freilich der letzte Teil hucusque Lacedmoniorum leges (!) dominatae sunt per annos CCCL zu Ol. 1, 1. Aber die Summe 350 hat doch auch er bewahrt; und diese fubrt von a. Abr. 916 - bei Hieronymus und beim Armenier das ${ }^{\circ}$. Jahr des Eurystheus - doch auf a. Abr. 1265 - $=01.7,1$ bei Hier., Ol. 7, 2 beim Armen. - Das ist 3 Jahre spüter als das echte Datum fur die Einsetzung des Ephorats 754/3 01. 6, 3 und erklärt sich durch die Herabschiebung von Eurysthens' 1. Jahr von a. Abr. 914 1103/2 auf a. Abr. 916 1101/0, die ihrerseits wieder mit der oben S. 426 besprochenen Herabschiebung von Eusebios' troisehem Datum 1184/3 auf 1182/1 durch den Interpolator zusammenhängt]. - So ist nach allem nur das Missverständnis erklärt, mit dem der Interpolator die Einsetzung des Ephorates als Abschaffung des Königtums gedeutet hat, nicht aber, warum er dann doch das letzte Königsjabr in 777/6, ea. 25 Jahre vor das erste Ephoratsjahr gesetzt bat. Vermutlich hängt das mit dem Ausfall von Menelaos' Regierung zusammen.

1) Die Lesung gebe ich nach der Neuvergleichnng des Steines, die mir durch Herrn von Hullers Gute vorliegt. Der Name des Eponymos fehlt hier wie Ep. 1 und 20. Dass es Kreon war, ist unzweifelhaft.

2) Nur trügender Schein ist es, was im ersten Augenblick für ScHwartz' Ansatz Kreons auf $682 / 1 \mathrm{zu}$ sprechen scheint, dass nämlich auf diese Weise die Königsherrschaft in Athen genau 900 Jahre dauert $(1582 / 1-683 / 2)$. S. Schwartz a. a. O. S. 55. Aber der Chronist selbst rechnet nicht so: er setzt Ep. 1 Kekrops' Beginn 1318 Jahre vor Diognetos, Kreon aber 420. Die Königsherrschaft dauert also bei ihm 898 oder im besten Falle 899 Jahre. Es ist ja allerdings klar, dass der Autor des im Marmor 
Euseb im Kanon das zweite Jahr giebt, so stützen sich diese beiden von einander unabbängigen Zengnisse gegenseitig so gut, dass wir 683/2 für Kreon als sicher bezeichnen düren. Das Zengnis des Marmors sichert das Datum des Kanons gegen den Verdacht der Interpolation; das Datum des Kanons erlaubt die Entscheidung zwischen den beilen möglichen Tahren des Marmors. Nicht unwichtig ist, dass das durch Euseb indizierte Jahr 683/2 für den Parier die Zählung von 2632 ergiebt (Computus B), die in dem ersten Teile des Marmors bis Ep. 67 zwar nicht die allein herrschende ist, ${ }^{1}$ ) wohl aber die überwiegende.

683/2 $=01$. 24, 2 also ist das Epochenjahr der attischen Liste. Daran haben wir ihren ersten Teil zu messen. Wir konstatieren danach, dass sich Pausanias mit seinen Angaben hier, wie bei anderer Gelegenheit, um gerade eine Olympiade geirrt hat. ${ }^{2}$ ) Bedenklicher steht es um Dionys, dessen chronologische Angaben sonst eine recht grosse Zuverlässigkeit aufweisen. Ol. 7, $1752 / 1$ ist nicht, wie er sagt, las erste, sondern das zweite Jahr des Charops. Ein Schreibfehler ist wenig wahrscheinlich; eher lässt sich an ein einfaches Versehen bei Benutzung von Tabellen denken, die römische Jahre mit attischen und Olympiadenjahren glichen, oder an einen Rechenfehler.i) Das bleibt bedenklich; aber gegenüber

vorliegenden Systems so gerechnet hat, wie Scrwar'rz meint, und die Königsherrschaft auf rund 900 Jabre $=27$ yev\&ci veranschlagt hat (vgl. dazu S. 432, 1). Wenn die Differenz der Epochenzablen trotzdem nur 898 (899) beträgt, so hat der betreffende Chronologe entweder Kreons Jahr mit eingerechnet oder aber, was ich glaube, wir haben hier eine der ublichen pragmatisierenden Korrekturen um 1 oder 2 Jahre vor

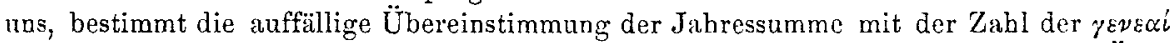
zu verstecken, der ganzen Liste also eineu mehr bistorischen Anstrich zu geben. Über diese "Anwendung der bei den Chronographen so uberaus beliebten beweglichen eins" vgl. z. B. R. Schubert, Geschichte der Könige von Lydien 1884, S. 11 ff. Keinesfalls also darf die angenommene Rundzahl 900, die, wie gesagt, ter Chronist selbst gar nicht liefert, uns veranlassen, Kreon auf 682/1 anzusetzen.

1) Diese Frage hier erschöpfend zu erörtern, wurde zu weit fuhren. Es genuge der Hinweis auf Ep. 37. 38, aus denen sich die Rechnung von 26t/3 (Вовскпs Computus A) nun einmal durch keine Kunststicke vertreiben liisst. Ep. 37 wird der Archon Simon(ides) 327 Jahre vor Diognetos angesetzt, $264 / 3+327=591 / 0.263 / 2+327$ $=590 / 89$. Aber 590/89 ist durch Aristoteles ' $A \vartheta \pi$. XIll 1. 2 als Anarchiejahr erwiesen. Für Simou bleibt also nur 591/0. Vgl. Philol. Unters. XVI S. 169 f. - Ep. 38 wird Damasias 318 Jahre vor Diognetos angesetzt, 9 Jahre nach Simon. Nur sein erstes Jahr 582/1 $(264 / 3+318=582 / 1)$ kann in Frage kommen, da es ein Pythien- also drittes Olympiadenjahr sein muss. Diese beiden Epochen genigen, die jetzt fast allgemein angenommene Ansjeht von einer aussehliesslichen Herrschaft des Computus B im ersten Teile des Marmors zu widerlegen. In derselben Partie aber stehen noch mehrere nach Computus A berechnete Epochen; so sicher Ep. 40, wahrscheinlich auch 41, 42, 44. Ep. 39, 43 sind die Epochenzahlen zerstört.

2) Vgl. S. 410, 2.

3) Unmöglich scheint mir die Erklärung durch verschiedenen Jahresanfang, wie sie auch Bosolt, Gr. G. II" S. 133 Anm. nach Gezzer giebt. Das im Herbst beginnende makedonische Jahr kann, wenn es mit einem Archonten geglichen werden 
dem Zeugnis des Marmors, das durch Kastor-Euseb bestätigt wird, kann Dionys keinesfalls aufkommen. ${ }^{1}$ )

Gehen wir nun dazu über, die Liste Kastors auf der sicheren Grundlage Kreon $=01.24,2$ 683/2 zu rekonstruieren. Auch ihr Beginn, das erste Jahr des Kekrops $=$ a. Chr. 1556/5, steht fest. Euseb giebt dieses Datum sowohl in dem Auszuge aus Kastor, Chron. I 187, 28 Simul colliguntur Atheniensium a Kekrope, qui Diphyes nominabatur, usque ad primam olompiadem, anni DCCLXXX. $(776 / 5+780=1556,5)$, wie in der Einleitung des Kanons p. 6, 17. 9, 28. Richtig setzt er auch in den fila regnorum Kekrops 1. Jahr =a. Abr. $461=$ a. Chr. 1556/5 (Hieronymus). Scnwartz hat dieses Anfangsdatum der Liste, die das Rückgrat der Chronologie auch in Kastors Chronik bildete (Königslisten S. 95), durch die entsprechenden Anfangsjahre anderer von der attischen abhängigen Listen noch weiter gesichert. Dennoch beginnen wir unsere Rekonstrukion besser nicht mit den Erechtheiden, sondern mit dem letzten und wichtigsten Teile der Fasten, weil dieser sicher begrenzt wird einerseits durch die überlieferte Gleichung eines attischen Königsjahres mit Ol. 1, 1 776/5 andrerseits durch das nun feststehende Jahr Kreons 683/2.2)

Euseb. Chron. I 187, 24 heisst es: Vigesimus nonus Eskhiles Agamestoris (filius) annis XXIII. Cuius duodecimo anno prima olompias con-

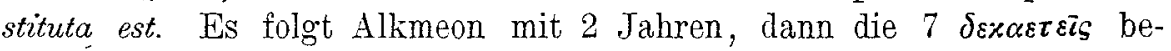
ginnend mit Charops, deren jeder 10 Jahre regiert. Das ergäbe:

soll, nur dem im Juli des gleichen julianischen Jahres beginnenden attischen Jahre gleichgestellt werden, nicht dem im Juli des Folgenden beginnenden. Da würden sie sich ja nur mit einem Vierteljahr decken. Nur die Gleichung Kreon attisch Juli 683/2 $=$ makedonisch Herbst 683/2 ist denkbar, nicht aber Herbst 683/2 = Juli 682/1.

1) Ungangbar ist der, übrigens scbon von Вовскн, $C I G$. II S. 332 eingeschlagene Weg Toprfers, Beiträge S. 287, es sei nicht wahrscheinlich „dass jeder der sieben $\delta \varepsilon \varkappa \alpha \varepsilon \tau \varepsilon \tau_{S}$ seine zehu Jahre abregiert haben soltte." Denselben Versuch, die Diskrepanz zwischen Kastor und Dionys auszugleichen macht auch UNGER, Troische Ära des Suidas 1885 S. 71. Alle drei denken natürlich an Hippomenes, weil unter ihm eine Verfassungsänderung stattgefunden haben soll. Aber dieser bistorische Grund kann auch abgesehen von seiner methodischen Unrichtigkeit fur die chronographische Betrachtung nicht in Rechnung kommen. Fưr die Rechnung der Chronographen sind uns 70 Jabre als Dauer der Dekaetie bezeugt nicht nur von Kastor b. Euseb. Chron. I 183, 5 deinde per decennium principatum tenebant, qui erant VII numero, tenueruntque annos LXX. (sämtliche aus Eusebios geflossene Listen stehen damit in Übereinstimmung); sondern auch ron Velleius Paterc. I 8, 3: coeperuntque in denos annos creari; quae consuetudo in annos septuaginta mansit. Die historische Untersuchung wird zeigen, warum der Chronograph, dem Kastor seine Liste verdankt, gar nicht anders konnte, als jedem $\delta \varepsilon x \alpha \varepsilon \tau \eta \dot{s} s$ auch wirklich seine 10 Jahre zu geben, wenn er nicht uberhaupt auf die Aufstellung einer Liste mit festen Jahreszahlen verzichten wollte.

2) Das hat Töprefer a. a. O. richtig erkannt. Denn nur so ist es möglich, zu sicheren Resultaten zu gelangen, die sich ToprFer selbst freilich durch seinen Ansatz Kreons unmöglich gemacht hat. 
Ol. $1,1776 / 5=$ Aischylos 12. Jahr

$$
\begin{aligned}
& " 3,4765 / 4=\quad " 23 . \\
& \text { " 4, } 1764 / 3=\text { Alkmeons } 1 \text {. " } \\
& \text { " 4, } 3762 / 1=\text { Charops } 1 . " \\
& 692 / 1=\text { Kreon }
\end{aligned}
$$

Das ist unmöglich. Sehen wir uns also den zweiten Zeugen für diesen Teil der Liste an. Nach dem Barbarus p. 41 a füllt 0l. 1, 1 in das 2. Jahr des Aischylos. Aber Alkmeon hat bei ihm nicht 2, sondern 10 Jahre und steht unter den $\delta \varepsilon \approx \alpha \varepsilon \tau \varepsilon i \bar{s}$. Also:

Ol. 1, $1776 / 5=$ Aischylos 2. Jahr

$$
\begin{aligned}
& \text { " } 6,2755 / 4=\quad " 23 \text {. " } \\
& " 6,3754 / 3=\text { Alkmeons } 1 \text {. " } \\
& " 8,4745 / 4=\quad " 10 . \\
& \text { "9, } 174411=\text { Charops } 1 . \\
& 6743=\text { Kreon. }
\end{aligned}
$$

Das geht wieder nicht. Hier setzt nun ScHwartz mit einer geistvollen Kombination ein: dass der Barbarus Alkmeon unter die $\delta \varepsilon x \alpha \varepsilon \tau \varepsilon i s$ stellt, ist richtig; aber ebenso richtig stellt ihn Chronic. I unter die diç Biov

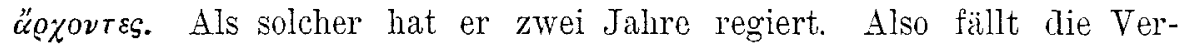
fassungsänderung in seine Regierung, die im ganzen 12 Jahre dauert. Die Liste Kastors lautete also:

Ol. 1, $1776 / 5=$ Aischylos 12. Jahr

$$
\begin{aligned}
& \text { "3,4765/4=" }=23 \text {. " } \\
& \text { "4, } 1764 / 3=\text { Alkmeons } 1 . " \\
& " 4,3762 / 1=\quad \text { " } \quad \text {. } \quad \text { " Verfassungsänderung. } \\
& \text { " } 6,4753 / 2=\quad " 12 \text { " } \\
& \text { " } 7,1752 / 1=\text { Charops } 1 . \\
& 682 / 1=\text { Kreon. }
\end{aligned}
$$

Ich will die Einwände, die sich auf den ersten Blick gegen eine derartige Annahme erheben lassen, hier nicht vorbringen. Sie werden im Laufe unserer Untersuchung noch vermehrt werden und wir thun besser, die Frage nach der historischen Richtigkeit der jetzt allgemein angenommenen Verfassungsänderung unter Alkmeon .oder vielmehr nach Beseitigung Alkmeons, auf den historischen Teil unserer Betrachtungen zu versparen. Da werden wir uns fragen, ob es wirklich, wie ScHwarTz behauptet, "als Überlieferung der attischen Chronik anzusehen ist, dass der letzte lebenslängliche Archont auch der erste zehnjährige war." Für jetzt genügt es uns zu konstatieren, dass unsere Überlieferung ausdrücklich Charops als ersten $\ddot{\alpha} \propto \chi \omega \nu \delta \varepsilon \chi \alpha \varepsilon \tau \eta \dot{s}$ nennt und die Zahl der befristeten Beamten auf sieben angegeben hat. ${ }^{1}$ ) Kastor

1) Velleius I 2, 2 sed hic (Medon) insequentesque archontes usque ad Charopem, dum viverent, eum honorem usurpabant. I 8,3 tum Athenis perpetui archontes esse 
kann nichts anderes behauptet haben. Es wäre doch zu merkwürdig; wenn wir annehmen müssten, dass das von Scrwartz aufgestellte Fundamentaldatum so ganz aus der Überlieferung verschwunden sei, während es einem Kastor bekannt war. Die bei Velleius vorliegende von Kastor jedenfalls mabhängige Tradition') berichtet aber die Entwicklung der königlichen Gewalt in Athen nicht anders als Euseb d. h. Kastor. MLit Interpolationen aus Eusebs Kanon können wir das doch nicht erklären. Aber noch seltsamer ist es, dass in den von Kastor abhängigen Listen gerade dieses Fundamentaldatum wieder bis zur Unkenntlichkeit verschleiert wird. Hätte Kastor in seiner Liste Alkmeon so berechnet, so bätte er die entsprechende Notiz gemacht und diese wäre uns wie die analogen Beischriften über die Dauer der einzelnen Herrscherfamilien, über die Einsetzung der Olympien zur Zeit des Aischylos, über die Einführung des Jahresamtes in 01. 24 und den ersten Jahresbeamten eben durch Euseb erhalten. Hier hätte der Interpolator gewiss nicht eingesetzt. Das konnte er bequemer haben. Ja, wenn der Barbarts Alkmeon 2+10 Jahre gäbe oder auch in einem Posten 12, so wäre Sorwartz Lösung immerhin wahrscheinlich. Da er ihm aber nur 10 giebt, dagegen die 2, die er in den übrigen Zweigen der Eusebischen Überlieferung hat, ausfallen lässt, so können wir hinter dieser Abweichung unmöglich profunde Gelehrsamkeit suchen. Es ist eine einfache Verschiebung, die nicht anders zu beurteilen ist, als die von SoHwartz selbst ganz richtig angesehene in den Zahlen der Erechtheiden oder die andere in der Reihe der lebenslänglichen Archonten. Dort ist der zweite Name (Kranaos) ansgefallen, die Regierungsdaten sind infolgedessen gegenüber den anderen Listen um je einen Platz hinaufgeschoben bis zum 8. Platz. Da steht kein Name, sondern nur eine Anmerkung. Ebenso ist bei den lebenslänglichen Thersippos vor Aischylos geraten und hat so dessen Zahl erhalten. Thersippos' eigene Zahl hat dafür die seines Vorgängers Archippos verdrängt. Nicht anders steht es mit Alkmeon. Der ist unter die $\delta \varepsilon x \alpha \varepsilon \tau \varepsilon \vec{c}_{S}$ geraten und hat so auch die Zahl X statt $\Pi$ bekommen. Der Grund aber der Verschiebung ist klar. Der Barbarus macht zu Aischylos eine umfangreiche Anmerkung, enthaltend die Angabe der Olympienstiftung und die Gesamtdauer des Königtums bis O1. 1. Dann fährt er fort: post Eschylum autem

desierunt, cum fuisset ultimus Alcmaeon, coeperuntque in denos annos creari: quae consuetudo in annos septuaginta mansit ac deinceps annuis commissa est magistratibus respublica. ex iis qui denis annis praefuerunt, primus fuit Charops, ultimus Eryxias, ex

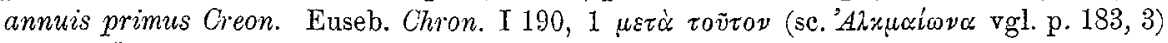

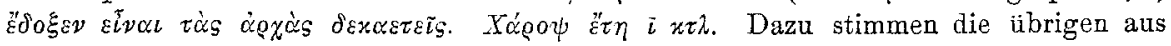
Eusebius geflossenen Listen.

1) Das zeigt schon der Umstand, dass Velleius a. a. O. das Archontat mit Medon beginnen lässt: huius flius Medon primus archon Athenis fuit, während Eusebius in Übereinstimmung mit dem Marmor die Oberbeamten bis Alkmeon $\beta \alpha \sigma i \lambda \varepsilon \tilde{l}_{S}$ nennt. Über die Verschiedenheit des Titels wird später zu sprechen sein. 


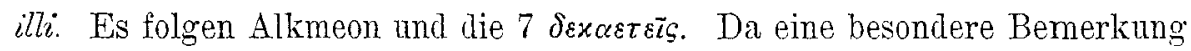
über die Einsetzung der Dekaetie fehlt, so war es beinahe notwendig, dass Alkmeons Zahl von den 7 folgenden Zehnen infiziert wurde. Der Barbarus hat also hier keinesweg's den echten Kastor bewahrt, nicht einmal einen Rest der echten Liste.

Suchen wir einen anderen Weg, der uns zum Ziele führt. Der Barbarus setzt doch nicht nur Alkmeon unter die $\delta \varepsilon x \alpha \varepsilon \tau \varepsilon i s$, er schreibt auch die Einrichtung der Olympienfeier nicht dem 12. sondern dem 2. Jahre des Aischylos zu. Schwartz erwähnt diese Diskrepanz mit keinem Worte, $\left.{ }^{1}\right)$ und doch beseitigt sie die scheinbare zehnjährige Lücke

1) Wenigstens nicht da, wo er sie hätte erwähnen mussen, nämlich bei der Rekoustruktion von Kastors Liste. S. 39 ff. stellt er Africanus' Fasten wieder her. Ogygos, der attische Autochthon, der mit der Exodos aus Ägypten gleichzeitig ist, wird von Africanus (bei Euseb. Praep. evang. X 10, 7. 10. 21. Synkell. S. 118, 21 ff.) 1020 vor Ol. 1, $1776 / 5$ gesetzt, d. h. 1796/5 (vgl. uber diesen Synchronismus die vorzugliche Auseinandersetzung von Schwartz S. $22 \mathrm{ffi}$ ), wieder nach Africanus (bei Euseb. Praep. ev. X 10, 14 vgl. Chron. I 181, $13 \mathrm{ff}$. Synkell. S. 131, $7 \mathrm{ff}$.) bleibt Attika danach dic

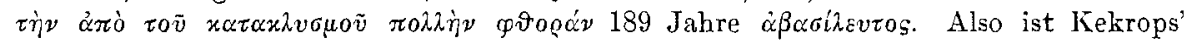
1. Jahr nach Africanus 1607/6. (Für das Beharrungsvermögen in Fragen chronologischer Natur ist es bezeichnend, dass Schwantz gegen die schon von Roude, Rhein. Mus. XXXVI 429, 1 = Kleine Schriften I 52, 1 widerlegte Zurickfubrung von Africanus' Zahlen fur Kekrops und Kranaos auf Philochoros noch einmal polemisieren muss. Für den, der die Stellen selbst auch nur fluchtig angesehen hat, ergiebt sich die Unbeweisbarkeit einer derartigen Zurückfuhrung auf den ersten Blick). Der Barbarus p. 41 a 18 giebt nun die Summe der Jahre von Kekrops bis Ol. 1, 1 auf 814 Jahre an, von Kekrops bis Kreon (p. 41 a 27) auf 907. Das gäbe fur Kekrops' 1. Jahr, da die Endpunkte $776 / 5$ und $683 / 2$ feststehen, 1590/89. Die Summen sind nicht Kastorisch. Als Africanisch erweist sie Schwartz durch die leichte Änderung von $\overline{\Omega I J}$ in $\overline{\Omega A A}$. $776 / 5+831=1607 / 6.907$ ist entstanden aus der bereits korrumpierten Zahl $814+93$ (776/5-684/3, letztes Königsjahr). Das ist alles klal. Die Hauptpunkte von Africanus' Rechnung sind damit wiedergewonnen. Wie aber diese Rechnung erweisen soll, „dass auch bei Africanus Aischylos’ 12. Jahr $=01.1,1$ war $^{\star}$, ist mir unverständlich. Die 93 Jahre von Ol. 1, 1 bis zur Einsetzung des ersten jährlichen Archonten setzen sich zusammen

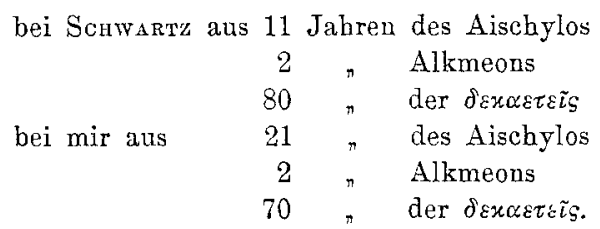

Bewiesen wäre also Scнwartz' Behauptung nur unter der Voraussetzung, dass Alkmeon unter die $\delta \varepsilon x \alpha \varepsilon \tau \varepsilon \tau_{\zeta}$ gehörte und Ol. 1, 1=13. Jahr [nicht 12.] des Aischylos wäre. Diese Voraussetzung aber soll ja gerade bewiesen werden. Auf diesem Wege kann Scuwdrtz die auch beim Barbarus erhaltene Gleichung 01. 1, 1= Aischylos' 3. Jahr doch nicht diskreditieren. Beim Barbarus liegen nach 01. 1, 1 die 21 Jahre des Aisehylos + 10 Alkmeons +70 der $\delta \varepsilon \varkappa \alpha \varepsilon \tau \varepsilon \tilde{\iota}_{S}=101$ Jahre, also $8 \mathrm{mehr}$ als möglich. Diese 8 uberschussigen Tahre sind dadureh entstanden, dass die urspriinglichen 2 Jahre des Alkmeon durch 10 verdrängt sind (oben S. 417). Interessant ist aber ein weiteres. 
im letzten Teile der Liste mit einen Schlage. Denn zum Barbarus stellt sich nicht nur Synkellos, ${ }^{1}$ ) sondern auch der Kanon, den wir bereits in der Ansetzung Kreons als zuverlässig erfunden haben. Hier heisst es (p. 78. 79): Secundo anno Aeschyli Atheniensizm iudicis prima olympias acta. Dazu aber tritt ein zweites: Hieronymus gleicht nicht etwa Ol. 1,1 mit Aischylos' 2. Jahr, sondern mit seinem dritten. Also

beim Armenier: a. Abr. $1240=0$ I. $1,1=$ Aischylos' 2. Jahr.

bei Hieronymus: a. Abr. $1241=0 \mathrm{l}$ 1, $1=$ Aischylos' 3. Jahr.

Zu Hieronymus aber stimmt Synkell. p. 368, 8 ed. Bonn., der sich ganz

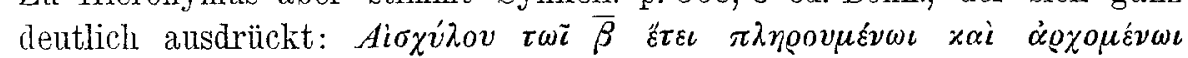

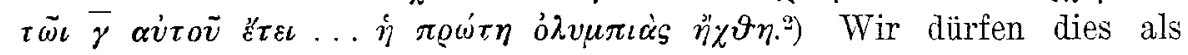
Ansatz Eusebs ansehen. Die Diskrepanz aber ist wichtig: sie lehrt uns zweierlei:

1. Die 23 jährige Regierung des Aischylos war durch die Olympienstiftung in zwei Teile zerlegt, 2+21. Zwei Jahre lagen vor Ol. 1, 1. Dieses selbst ist gleich dem dritten. Der ungenaue Ausdruck in der begleitenden Notiz, in der für das $\pi \rho \eta_{\gamma}$ O1. 1, 1 eingetreten ist, ist leicht erklärlich und hat seine Analogieen. ${ }^{3}$ )

2. Hier scheint mir die Erklürung für die falsche Olympiadengleichung des Armeniers gewonnen. Der ungenaue Ausdruck, der für Hieronymus weitere Folgen nicht hatte, da er sich der Gleichung Ol. 1, 1 $=$ Aischylos' 3. Jahr bewusst blieb, führt beim Armenier zur Herauf-

Scrwartz giebt ganz mit Recht als Dauer der Könige und $\delta \varepsilon x \alpha \varepsilon \tau \varepsilon \tilde{i}$ bis Kreon nach Africanus 924 Jahre. Das aber ergiebt von dem von Schwartz selbst festgestellten und sicher richtigen Anfangsjahr der Liste $1607 / 6$ gerechnet, als letztes Königsjahr 684/3, fur Kreon 683/2. Das ist das von mir für Kastor-Euseb berechnete Datum, das im Kanon erhalten ist und zu dem das Marmor stimmt. Aber wie stimmt es zu dem vou Schwantz berechneten Jahre 682/1?

1) S. 368,8 der Bonner Ausgabe, wo $\bar{\imath}$ aus dem $\bar{\beta}$ der Handschriften in peius korrigiert ist.

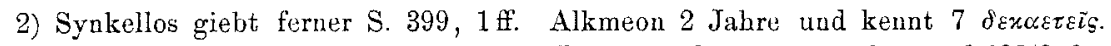
Das führt auch für ihn, da er Aischylos' 3. Jahr mit 01. 1, 1 gleicht, auf 683/2 fur Kreon. Diesen selbst bestimmt er in einer seltsam verwirrten Stelle S. 400, $4 \mathrm{ff}$. so:

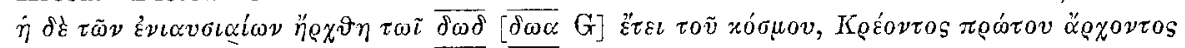

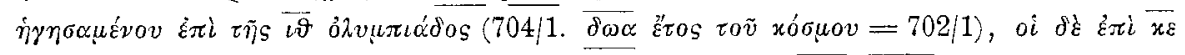

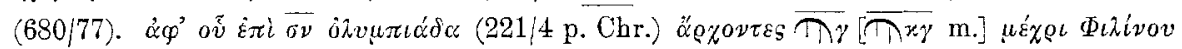

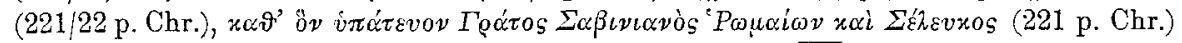

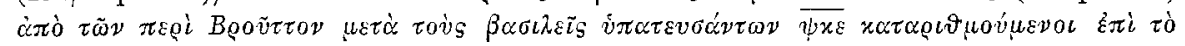

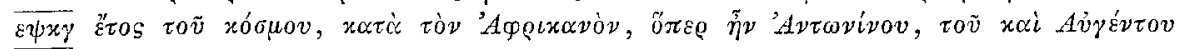

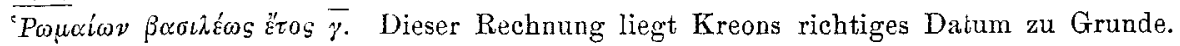
Woher die 19. und die 25. Olympiade kommen, weiss ich nicht. Sonst vgl. uiber die Stelle Gezzer, Africamus I S. 50, Sciwarmz a. a. 0. S. 24.

3) Vgl. Philol. Unters. XVI S. 78. 97, 9. 
schiebung von 01. 1, 1 in Aischylos' 2. Jahr. Aischylos' 3. Jahr ist bei ihm wie bei Hieronymus (also anch bei Euseb selbst) gleich a. A.br. 1241; also kommt 01. 1, 1 in a. Abr. $1240=$ Aischylos' 2. Jahr. Mrir scheint diese Erklärung einfach, weil sie aus den von Eusebios selbst Ol. 1 beigeschriebenen Notizen gewonnen ist. ${ }^{1}$ )

Stellen wir jetzt den letzten Teil der Liste auf:

$$
\begin{aligned}
& \text { Ol. } 1,1776 / 5=\text { Aischylos' 3. Jalır } \\
& \text { " } 6,1756 / 5=\text { " } 23 \text {. , } \\
& \text { "6, } 2755 / 4=\text { Alkmeons } 1 . \quad \text {, } \\
& \text { "6, } 4 \text { 753/2 }=\text { Charops' } 1 . \quad \text { " } \\
& \text { "24, } 2683 / 2=\text { Kreon. }
\end{aligned}
$$

Das ist die Probe anf das Exempel. Wir haben nichts geändert. Alkmeon

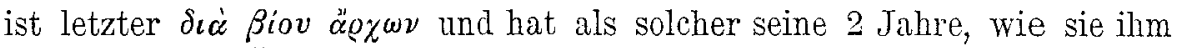
die Eusebische Überlieferung einstimmig giebt. Erster $\delta \varepsilon \varkappa \alpha \varepsilon \tau r_{r}^{\prime} S$ ist nach Eusebs formellem Zeugnis Charops; und Kreon kommt in 683/2, das durch den Parier und den Kanon gegebene Jahr. Wenn Chronic. I Ol. 1, 1

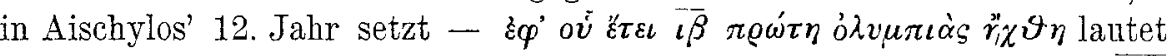
der griechische Text - so hat schon Scaliazr die Dittographie $\tilde{\varepsilon}_{\varepsilon \varepsilon \iota} \overline{[\imath] \beta}$ als solche erkannt. Bewiesen wird sie durch das einstimmige Zeugnis der Parallelüberlieferung, nämlich des Barbarus, des Synkellos und des Kanons.

Damit ist, glaube ich, der letzte und wichtigste T'eil der Liste gegen jede Anfechtung gesichert. $O b$ sich für die vorhergehenden Partieen ein gleich reinliches Resultat ergeben wird, erscheint zunächst fraglich. Es ist oben bemerkt, dass das Anfangsjahr der Liste 1556/5 feststeht, dass Euseb selbst den Abstand des Anfangsjahres von Ol. 1, 1 auf 780 Jahre angiebt und dass im Kanon richtig Kekrops' 1. Jahr mit a. Abr. 461 (=1556/5 bei Hieronymus) geglichen ist. Die Gesamtsumme der Regierungszahlen der Könige, lebenslänglichen und zehnjährigen Archonten ist also $873(1556 / 5-684 / 3)$. Diese Summe wird folgendermassen verteilt:

1) Unter diesen Beischriften ist die über den Synchronismus von OI. 1, 1 mit dem Könige Joatham ron Juda eine freche Interpolation (Schwartz S. 33); die über den Abstand von 01. 1, 1 bis Troias Fall - 405 Jahre - ist wenigstens in der Zahl interpoliert; denn diese setzt das um zwei Jahre herabgeschobene troische Datum voraus. Eine wirkliche Gleichung ist nur die von Ol. 1, 1 mit dem attischen Königsjahr. Auch sie zeigt, wie die unten zu besprechende von Troias Fall mit einem attischen Jahr, die ganz exceptionelle Stellung der attiseben Liste in Kastors System. Die synchronistischen Handbücher, wie Kastor eines verfasst hat, haben als chronologisehes Rückgrat die Olympiadenjahre, mit denen die attischen Arehonten und die römischen Konsuln geglichen werden. Dieser Vorzug der attischen Liste, die ja auch wirklich die sicherste war, wirkt dann nach oben fort und verschafft den attischen Synchronismen ibre bevorzugte Stellung. 


\begin{tabular}{|c|c|c|}
\hline \multicolumn{2}{|c|}{ Cbron. I 181,33} & Kanon $\left.{ }^{1}\right)$ \\
\hline Erechtheiden: & 450 & 429 \\
\hline Melanthiden: & $52[1.58]$ & 58 \\
\hline 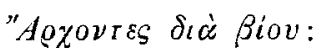 & $209^{2}$ & 316 \\
\hline "Aơ & 70 & 70 \\
\hline & $781[787]$ & 873 \\
\hline
\end{tabular}

Die Schlussummen der einzelnen Gruppen werden auch im Kanon angegeben, mit Ausnahme der "Ao\%. $\delta \iota \dot{c} \beta i o v$, die wir erst aus den Einzelregierungen berechnen müssen. In Chronic. I kennzeichnet sich die Zahl für die Melanthiden sofort als Schreibfehler ( $\overline{N B}$ für $\overline{N H I})$, da die Einzelposten p. $186,28-33$ richtig mit $37+21$ angegeben werden. Für die

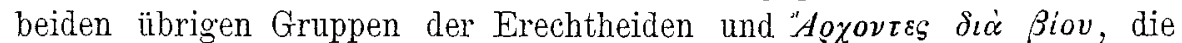
durch das sicher überlieferte Mittelstück der Melanthiden getrennt werden, müssen wir erst, wie es auch SoHwariz gethan hat, die Ubberlieferung der Einzelposten vorlegen.

\begin{tabular}{|c|c|c|c|c|c|c|c|}
\hline & & & hth & en: & & & \\
\hline & Eus & bios & Series & & Syn- & Xon & \\
\hline & Text & Kanon & Reg. & Barbarus & kell. & 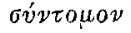 & 1. Jahr \\
\hline 1. $K \dot{x} \times 0 o \psi$ & $5[50]$ & 50 & 50 & 50 & 50 & 30 & $1556 / 5$ \\
\hline 2. Konvarós & 9 & 9 & 9 & $-[9]$ & 9 & 9 & $1506 / 5$ \\
\hline 3. 'Auff & $9[10]$ & 10 & 10 & $40[10]$ & 10 & 10 & $1497 / 6$ \\
\hline 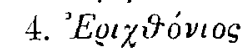 & 50 & 50 & 50 & $10[50]$ & 50 & 53 & $1487 / 6$ \\
\hline 5. $\Pi \alpha \alpha \nu i \omega \nu$ & 40 & 40 & \pm 0 & $50[40]$ & 40 & 40 & $1437 / 6$ \\
\hline 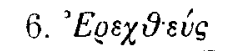 & 50 & 50 & 50 & $40[50]$ & 50 & 3 & $1397 / 6$ \\
\hline 7. $K \varepsilon \varkappa \rho о \psi \bar{\rho}$ & 40 & 40 & 40 & $53[40]$ & 40 & 43 & $1347 / 6$ \\
\hline 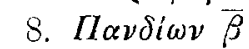 & 25 & 25 & 25 & $43[25]$ & 25 & 29 & $1307 / 6$ \\
\hline 9. Aijevís & 48 & 48 & 48 & 48 & 48 & 48 & $1282 / 1$ \\
\hline 10. $\Theta \eta \sigma \varepsilon u_{\varsigma}$ & 30 & 30 & 30 & 31 & 31 & 34 & 12343 \\
\hline 11. $M_{\varepsilon \nu \varepsilon \sigma \vartheta \varepsilon \dot{\nu} \varsigma}$ & 23 & 23 & 23 & 19 & 33 & 29 & \\
\hline 12. $\Delta \eta \mu о \propto \tilde{\omega} \nu$ & 33 & 33 & 33 & 35 & 23 & 33 & s.unten \\
\hline 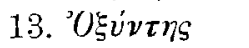 & 12 & 12 & 12 & 14 & 10 & 31 & S. 427. \\
\hline 14. 'Aq ${ }^{\prime} i \delta \alpha \varsigma$ & 1 & 1 & 1 & 1 & 1 & 一 & \\
\hline 15. Ovuoitns & 8 & 8 & 8 & 9 & 9 & 10 & \\
\hline & $383[429$ & 429 & 429 & $443[431$ & 429 & 402 & \\
\hline
\end{tabular}

1) A. Abr. 889 [die Notizen stehen bei Hieronymus s. a. Abr. 880. 887], Thymoites' letztes Jahr a. Chr. 1128/7: Castoris de regno Atheniensium: exponemus antem et Atheniensium reges cognomento Erecthidas a Cecrope difye usque ad Thymoeten, quorum omne tempus invenitur ann. CCCC XXVIIII [CCCC XLIX Armen.]. Post quos suscepit regnum Melanthus Andropompi fitius et huins filius Codrus, qui imperarunt simul annis LVIII. Ebenda a. Abr. 948 1. Jahr Medons. a. Chr. 1069/8 Codrus . interimitur bello Peloponnesiaco, in quo Erecthidarum regnum destructum est, quod CCCCLXXXVII [DLVIII Arm.] annis perseverabat. Ebenda a. Abr. 1264 Charops' 1. Jahr a. Chr. 753 2: Athenis principes qui usque ad mortem reipublicae praeerant desierunt et in I cunos magistratun consuetudo versa est, regnavitque primus Charops filius Aeschyli. 
In der Summe 429 stimmen Eusebs Text (nach den selbstverständlichen Besserungen von 5 in 50 und 9 in 10), Kanon, Series Regum, Synkellos überein. Doch giebt Synkellos dem Theseus 31 statt 30, Thymoites 9 statt 8 Jahre, und gleicht das durch die 10 des Oxyntes statt 12 wieder aus. Es ist bemerkenswert, dass die ersten beiden Abweichungen immer vereinigt erscheinen. Synkellos, der Barbarus, das Xoovoyoaqciov oívtouov, die alle Theseus 31 Jahre geben (die 34 des Xuovoye. sind nur verschrieben), haben auch 9 für Thymoites (im Xoovoyo. mit dem $1 \mathrm{Jahr}$ des Apheidas zusammengezogen). Die Zahlen für Demophon und Menestheus haben bei Synkellos Platz gewechselt. Der Barbarus weist, wie Schwartz mit Recht bemerkt, Eusebs Liste anf, die nur durch die bereits oben erwähnte Verschiebung, entstanden durch Ausfall des Kranaos, in Unordnung geraten ist. Merkwürdig sind die Zahlen für Erechtheus und Kekrops, 53 und 43 statt 50 und 40 . Auch bei ihm haben Theseus 31, Thymoites 9 Jalire; aber der Überschnss wird nicht ausgeglichen, sodass die Gesamtsumme 431 um 2 Jahre höher ist. Menestheus giebt er 19 statt 23 Jahre, gleicht das aber durch die 35 des Demophon und 14 des Oxyntes statt 33 und 12 wieder ans.

Fïr die Ermittlung der kastorischen Zahlen bietet uns der Fall Troias einen festen Punkt. Es ist eines der schönsten Resultate von Schwartz' Untersuchungen, als Datum des Falles 1184/3 festgestellt und damit die Abhängigkeit Kastors von Eratosthenes-Apollodor erwiesen zu haben. Eine Fülle von falschen Konstruktionen fällt damit zu Boden. Kastor hat also, da das Anfangsjahr 1556/5 ebenfalls feststeht, Troias Fall ins 373. attische Königsjahr gesetzt. Es ist wichtig, dass er hierin mit dem Marmor Parium stimmt, nach dessen Epochenzahlen zwischen Troias Fall und Kekrops 1. Jahr 374 oder - da ein Jahr im Marmor kein Jahr ist - 373 Jahre liegen. Der Autor von Kastors Liste hat hier das alte in der Atthis aufgestellte Intervall auch in seine Liste übernommen. Nach Eusebs Text, Kanon, Series Regum und Synkellos ist das 373. attische Königsjahr das 21. des Menestheus. Aber im Kanon ist Troias Fall um 2 Jahre nach unten verschoben, von $1184 / 3$ in $1182 / 1,{ }^{1}$ ) vom 21. in das 23. und letzte Jahr des Menestheus, in das 375. attische Königsjahr. Nach dem Barbarus schliesslich, der Theseus 31, Menestheus 19 Jahre regieren lässt, coincidiert Troias Zerstörung und das 373. attische Königsjahr mit Demophons 1. Jahr; đ. h. wenn wir die 6 überschiessenden Jahre in den Regierungen des Erechtheus und Kekrops II. ansser acht lassen. Wie hat Kastor gerechnet? Nach Schwartz hat der Barbarus hier den echten Euseb und damit Kastor bewahrt; und diese Ansicht

1) Kanon a. Abr. $835=$ a. Chr. 1182/1 = Menestheus 23, Jahr [beim Armenier ist a. Abr. $835=$ a. Chr 1181/0. Hieronymus giebt ausdricklich das 375 . Königsjahr anj 1184/3 als troisches Datum Eusebs steht fest durch Praep. ev. X 9, 6. 
scheint eine gewisse Beglaubigung zu gewinnen, wemn wir sehen, dass von den zwei anderen Angaben, die Troias Fall auf ein attisches Jahr fixieren, die eine - es ist die des Lysimachos, der aber nur eine Vermittlerrolle spielt ${ }^{1}$ ) - wirklich Demophons 1 . Jahr giebt; freilich ist das Datum des Lysimachos zweimal überliefert, und der eine Zenge nennt das 4., der andere das 1. Jahr des Demophon. Aber die Wahrscheinlichkeit spricht für letzteres; denn wie es scheint, ist die Tradition in diesem Punkte schon selır früh dahin festgestellt, dass Troia im letzten Jahre des Menestheus gefallen ist (s. unten S. 424, 5). Wenn dafür Demophons 1. Jahr eintritt, so ist das eine unbedeutende Variante, die leicht zu erklären ist, während das 4. Jahr dieses Königs eine abweichende Tradition darstellen würde, für deren Entstehung sich keine Erklärung. finden lässt. Doch darüber später.

Kehren wir zu Kastor zurück. Auch hier scheint mir Scewair'z zu hastig vorgegangen zu sein und die anderen Möglichkeiten zu wenig berücksichtigt zu haben. Mit welchem Rechte vindicieren wir gerade Lysimachos' Datum dem Kastor? Diese ganze Konstruktion mit ihrem zufälligen Zusammentreffen ruht auf der Zahl 31 für Theseus. Ist dies die Zahl Eusebs? Oder ist sie ein einfacher Schreibfehler $\bar{A}$ für $\bar{A}$ ? Sieht man die analogen Zahlen für Erechtheus und Kekrops II. an, wo der Barbarus statt der sicher Eusebischen 50 und 4053 und 43 hat,

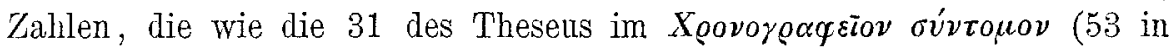
3 korrumpirt) wiederkehren, so wird man kaum geneigt sein, gerade den 31 Jahren für Theseus, trotzdem sie auch bei Synkellos erscheinen, einen besonderen Wert beizulegen. Jedenfalls lohnt es sich, anch einmal die anderen Möglichkeiten zu erwägen. Die von dem parischen Chronisten benutzte Atthis setzt Troias Fall in Menestheus' 22. Jahr, ${ }^{2}$ ) und wir sahen bereits, dass der Autor von Kastors attischer Liste das Intervall, das diese Atthis zwischen Kekrops und Troias Fall legte, geschont hat.

1) S. jetzt Rudtke, De Lysimacho Alexandrino (1893) S. 15f. - Die Stellen sind

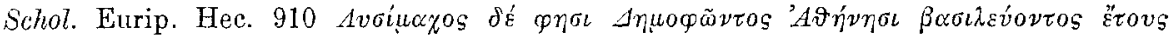

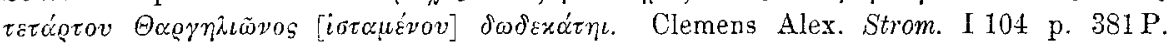

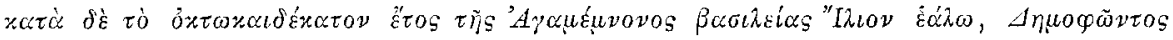

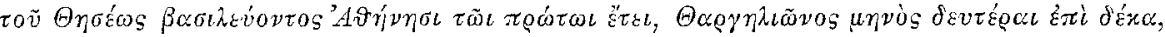

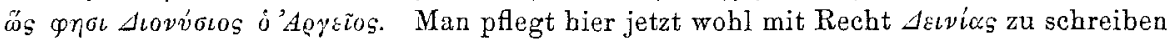
(s. uiber ibn Schwartz bei Pauly. Wissowa IV 2389f.). Dies Datum stand also in einer argivisehen Chronik, wie denn Clemens gleich darauf Hagias und Derkylos zitiert. Aber gehört der Synchronismus zwischen Agamemnons 18. und Demophons 1. Jahr auch dem argivischen Sammler? wahrscheinlich; denn Clemens stellt dem gegenüber $\tau \iota \nu \bar{\varepsilon} s$

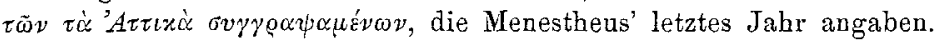

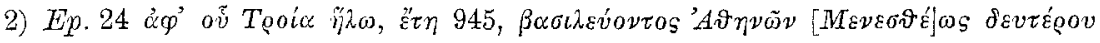

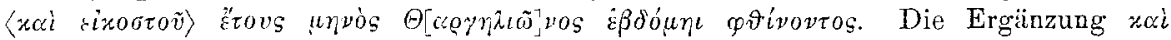
¿ixortó ist gesichert durch Ep. 29, wo der Auszug der Griechen gegen Troia in Menestheus' 13. Jahr gesetzt wird. 
Wenn wir nun bei Euseb. Chron. I 185, 16 lesen: Undecimus Menestheus ... cuius atate Ilium captum; wenn wir im Kanon das gleiche mit der Begründung finden: a primo anno Cecropis ... usque ad captivitatem Troiae et usque ad XYIII annim Menesthei, cuins Homerus meminit, anni CCCLXXV, so werden wir doch zweifelhaft, auf welcher Seite Kastor gestanden hat. Die Überlieferung, dass es Menestheus ist, der die Athener vor Ilion führt, beginnt mit Il. $B$ 552. $\Delta$ 327. $M$ 331. $N$ 690; und diese Überlieferung ist einheitlich. ${ }^{3}$ ) Das späte Eindringen der Theseussöhne :) hat an dieser Stellung des Menestheus nichts zu ändern vermocht. Lysimachos selbst bringt an anderem Orte eine Stelle der $\Pi_{\text {Eo }}$ is bei, in der die Theseiden genannt werden, aber Menestheus als $\pi$ ou $\mu \nu \nu$ $\lambda \alpha \tilde{\omega} \nu$ bezeichnet wird. ${ }^{3}$ ) Den Ansatz auf Demophons 1. Jahr aber hat er einer argivischen Chronik entnommen (s. S. 423,1). An derselben Stelle, an der Clemens Alexandrinus das von Lysimachos vermittelte argivische Datum überliefert (Strom. I 104 p. 381 P), bringt er dagegen die Meinung von

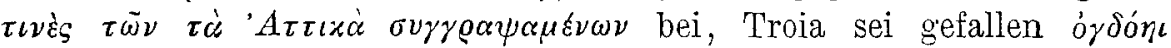

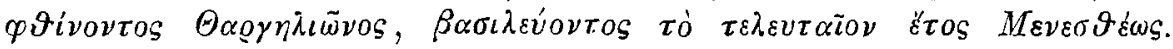
Kastor kann nichts anderes berichtet haben; seine Stellnng erhellt zu deutlich aus den citierten Notizen Eusebs. Es bliebe nur eine Möglichkeit: Troia ist nach der überwiegenden Ansicht im Thargelion, also im vorletzten Monat des attischen Jahres, gefallen. Hat Kastor vielleicht Menestheus noch in den letzten Tagen dieses Jahres sterben lassen, sodass ihm 118t/3 chronographisch schon als erstes Jahr. Demophons gelten musste? Dass er das nicht gethan hat, lehrt die weitere Notiz des Kanons: Menestheus moritur in Melo regrediens a Troia; post guem Athenis regnavit Demophon. Danach kann es keinem Zweifel unterliegen, dass Kastor Menestheus' Tod in das auf Troias Fall folgende Jahr 1183/2 gesetzt hat, ${ }^{4}$ ) dass Demophons 1 . Jahr also nicht $1184 / 3$ ist, sondern 1183/2. Denn auch darin ist die Überlieferung fest, dass Menestheus nicht mehr nach Athen zurückgekehrt ist. ${ }^{5}$ ) Kastor hat genau so berichtet, wie

1) Vgl. u. a. [Apollodor.] Biblioth. ep. III 11. V 22. Pausanias I 23, 8 (die Nachbildung des bölzernen Pferdes auf der Akropolis mit Menestheus, Teukros und den Theseussöhnen, die schon Aristophanes kennt) s. Loewy, Inschriften Griechischer Bitdhauer No. 52.

2) Vgl. darüber Widanowitz, Plit. Unters. I 101, 8. 125; Tópfrer, Quccestion. Pisistrat. S. $72 \mathrm{ff}$.

3) Schol. Euripid. Troad. 31.

4) Schwartz' Verlegenheit gegenüber diesen Thatsachen zeigt sich in seinen Worten S. 46f: „Der Fall Troias soll in das letate Jahr des Menestheus fallen, was nicht ensebianisch ist [warum nicht?], aber die Meinung des Africanus gewesen sein kann: ob es wirklich so gewesen ist, weiss ich nicht".

5) Vgl. ausser den Chronographen [Apollodor.] Biblioth. ep. VI $15 \mathrm{~b}$ (Tzetzes

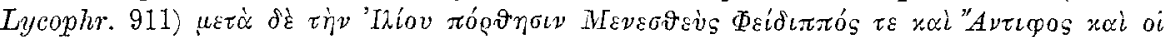

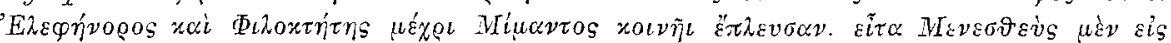


Dionys. Hal. AR. I 63 in der ausführlichen chronologischen Erörterung über das Datum von Troias Fall: er giebt dafür wie die Atthis die

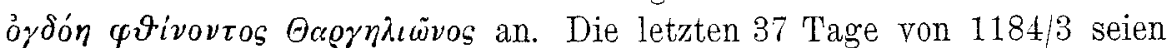

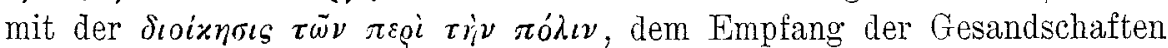
$\tau \bar{\omega} \nu$ àq $\varepsilon \sigma \tau \eta * o ́ r \omega \nu$ und der Schliessung von Verträgen vergangen. Tü

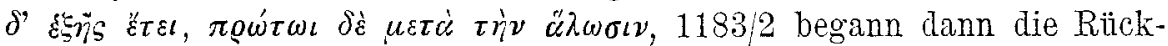
fahrt. Auf dieser ist Menesthens gestorben. Der Grund der Übereinstimmung ist klar: Kastor wie Dionys folgen der EratosthenischApollodorischen Chronologie. Eratosthenes aber benutzt, wie das Tagesdatum zeigt, die Atthis. Doch über Eratosthenes als Quelle Kastors unten.

Kastor also hat Troias Fall in das letzte Jahr des Menestheus gesetzt. Wit dieser Thatsache musste der Interpolator des Kanons rechnen, als er Troias Fall von $1184 / 3$ auf $1182 / 1$ herunterschob. Wollte er nicht die ganzen Bestimmungen, mit denen Euseb dieses wichtige Datum umgeben hatte, streichen - und das konnte er bei einem so festen Synchronismus eben nicht - so musste auch in dem interpolierten filum Troias Fall in Menesthens' letztes Jahr kommen. Das liess sich nur erreichen, wenn er die Dauer der vorhergehenden 11 Regierungen um 2 Jahre verlängerte. Eine derartige Interpolation war leicht zu bewerkstelligen; aber wo sie vorgenommen ist, lässt sich nicht ohne weiteres sagen. Doch glaube ich, dass uns auch hier der Weg gewiesen ist durch die auffällige Gleichheit, die wir zwischen der vortroianischen Chronologie des Marmors und Kastors bereits konstatieren konnten. Bei beiden fiel der Fall Troias in das 373. attische Königsjahr, bei beiden in das Ende von Menestheus' Regierung. Wir werden unten sehen, dass, soweit der Zustand der Marmorchronik ein Urteil erlaubt, auch die Dauer der Einzelregierungen von Kekrops bis Aigeus in der Atthis des Pariers die gleiche war wie bei Kastor. Die erste Differenz scheint sich in Theseus' Regierung zu finden. Theseus' 1. Jahr wird durch Ep. 20 auf 1259/8 (1258/7) fixiert, Nenestheus' Beginn durch Ep. 23 auf 1230/29 (1229/8); d. h. Theseus regiert nach dem Parier 29, nach Euseb 30 Jahre. Zweitens aber setzt der Chronist Troias Fall in Menesthens' 22. Jahr. Da unsere Überlieferung ihn entweder in Demophons 1. Jahr setzt - so Deinias der Argiver, den Lysimachos wiedergiebt - oder in Menestheus' letztes, eben weil dieser auf dem Rückwege von Troia nach Athen starb, da Clemens als Vertreter dieses letzten Ansatzes $\tau \iota \nu \dot{s} \varsigma \tau \tilde{\omega} \nu$ $\tau \dot{\alpha}{ }^{2} A \tau \tau \iota \varkappa \dot{\alpha} \sigma v \gamma-$

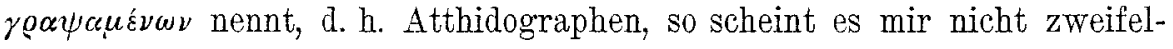

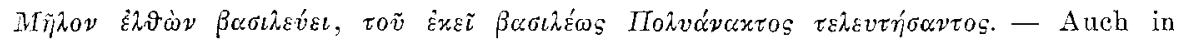
Italien und Spanien werden Grundungen des Menestheus genannt (Strabon III 140, VI 261). Die Überlieferung uber seine Wanderungen wird verschieden gewesen sein; aber nach Athen ist er nicht zuruckgekehrt. 
haft, dass das 22. Jahr des Menestheus, in das er 'I'roias Fall setzt, dem Chronisten eben als das letzte dieses König's galt, der nach der interpolierten Liste Eusebs 23 Jahre regiert. ${ }^{1}$ ) Die Sache scheint mir also so zu liegen, dass der Interpolator des Kanons, der Kastors von Euseb acceptiertes troisches Datum 1184/3 auf 1182/1 heruntergeschoben hat, die so erforderlichen zwei Königsjahre mehr dadurch gewonnen hat, dass er den beiden letzten vortroianischen Regierungen des Theseus und Menestheus je 1 Jahr zuschlug. Auf diese Weise behielt er den richtigen Anfang der Liste ebenso bei, wie den Synchronismus zwischen Troias Fall und Menestheus' letztem Jahr. Dass der Fall ins 375. statt wie bei Kastor ins 373. attische Königsjahr kam, war ein geringes Unglïck. Die Interpolation selbst ist anch in die Chronik eingedrungen; aber nicht nur in diese, sondern auch in die Excerpta Barbari. Da Anfang und Ende der Königsliste, $1556 / 5$ und $684 / 3$, feststanden, anch vom Interpolator des Kanons, wie wir sahen, festgehalten sind, so muss er die nachtroianischen Regierungen um eben die zwei Jahre gekürzt haben. Wo das geschelien ist, lässt sich wieder nicht ohne weiteres sagen. Sehen wir die Liste an, die Schwartz als kastorisch aufgestellt hat, so finden wir als 1. Jahr des Melanthos, mit dem ein neues Geschlecht zu herrschen

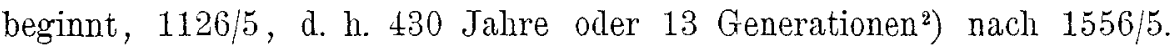
Dies Datum sieht echt kastorisch aus. Dazu kommt, dass sich so der Schreibfehler in der Gesamtzahl der Erechtheiden leicht erklärt. Schon Schwartz hat Eusebs $\overline{Y N}$ in $\overline{Y A}$ korrigiert. Das heisst: der Interpolator hat, was auch an sich wahrscheinlich ist, die zwei Jahre, die er Theseus und Menestheus zuviel gab, bei den Königen vor Melantlos wieder gestrichen, die Gesamtsumme der Erechtheiden also nicht angetastet.

Ganz sicher ist ein derartiger Schluss natürlich nicht. Denn es ist nicht ausgeschlossen, dass Kastor den Erechtheiden eben nur 428 Jahre gegeben hat - diese kleinen Änderungen, die den Ursprung der Regierungsdauern aus einfacher Multiplikation der Zahl der Generationen mit $33 \%$ verdecken sollen, sind in derartigen Listen häufig genug - und die Korruptel von $\overline{Y K I}$ in $\overline{Y N}$ ist auch nicht unerklärlich. Dann hätte

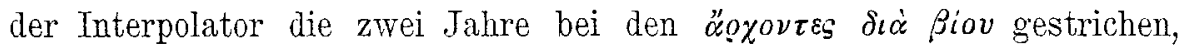
wo die Überlieferung stärkere Diskrepanzen zeigt. Ich bemerke das nur, um za zeigen, dass die Einzelregierungen von Troias Fall bis 01. 1 mit absoluter Gewissheit sich nicht wiederherstellen lassen. Aber wie

1) Bisher pflegte man ebon aus Eusebs interpolierter Liste zu schliessen, dass der Parier 'Troias Fall in Menestheus' vorletztes Jahr gelegt habe, ein Datum, dass gar keinen Sinn hat.

2) Die Liste enthält freilich bis Thymoites 15 Namen; aber Demophon als Sohn des 'Theseus bildet mit Menestheus eine $\gamma \varepsilon \nu \varepsilon a ́$; ebenso Thymoites mit Apheidas als Söhne des Oxyntes. 
gesagt, das Datum 1126/5 für Melanthos Beginn macht den Eindruck des echt kastorischen. Kastor hätte dann als Gesamtdauer der Könige von Demophon bis Thymoites 57 . Tahre berechnet (1183/2-1127/6), der Interpolator des Kanons nur 55 (1181/0 -1127/6). Dem entspricht die Überlieferung:

\begin{tabular}{lccc} 
& Euseb. Text Kanon. & Barbarus. & Synkellos. \\
Demophon & 33 & 35 & 33 \\
Oxyntes & 12 & 14 & $10[12]$ \\
Apheidas & 1 & 1 & 1 \\
Thymoites & 8 & 9 & 9 \\
\cline { 2 - 4 } & 54 & 59 & $53[55]$
\end{tabular}

Direkt auf die verlangte Summe führen sowohl der Barbarus, dessen 4 überschiessende Jahre durch die 4 bei Vienestheus fehlenden ausgeglichen werden (er giebt Menestheus nur 19 statt 23), wie Synkellos; denn die 10 Jahre des Oxyntes sind offenbar Korruptel aus 12. Diese Übereinstimmung zeigt, dass für Thymoites 9 Jahre das richtige sind.

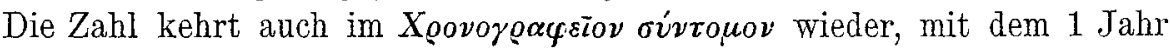
des Apheidas zu 10 zusammengezogen. Der Grund, aus welchem der Interpolator Thymoites' Regierung um 1 Jahr gekürzt hat - im Kanon ist daher nicht 1126/5, sondern 1127/6 1. Jahr des Melanthos. Das zeigt, dass wirklich der Interpolator am Werke war - ist freilich schwer abzusehen. Kastors Daten können wir für die 4 Könige von Demophon bis Thymoites bis auf zwei Jahre genan natürlich nicht wiederherstellen. Denn da die Interpolation auch die Excerpta inficiert hat, fehlt uns jedes Mittel, zu konstatieren, welche der vier Regierungen um die 2 Jahre gekürzt ist. Als kastorisch ist also nur festzustellen:

$$
\text { Demophon-Thymoites } 57 \text { Jahre } 1183 / 2-1127 / 6
$$

es folgen die beiden Melanthiden, deren Zahlen unbestritten sind:

$\begin{array}{llll}\text { Nelanthos } & 37 & \text { Jahre } & 1126 / 5-1090 / 89 \\ \text { Kodros } & 21 & 1089 / 8-1069 / 8\end{array}$

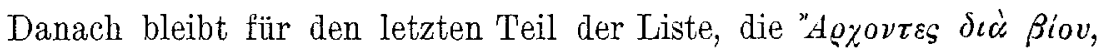
die begrenzt wird durch Medons 1. Jahr 1068/7 und Alkmeons letztes $754 / 3$ (s. oben S. 420), eine Dauer von 315 Jahren.

Die eingestandenermassen korrupte Summe bei Euseb. Chron. I 183, 4 ist danach zu korrigieren; $\overline{C \Theta}$ in $\overline{T I E} \cdot{ }^{1}$ ) Die Überlieferung der Einzelregierungen ist diese:

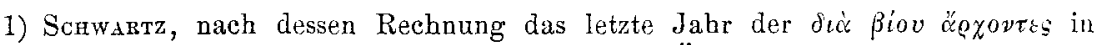
$763 / 2$ fällt, muss natürlich $\bar{\sigma} \bar{\vartheta}$ in $\overline{\tau_{\varsigma}}$ korrigieren. Die Änderung ist nicht leieht. 


\begin{tabular}{|c|c|c|c|c|c|c|c|c|}
\hline & Text & $\begin{array}{l}\text { ebios } \\
\text { Kanon }\end{array}$ & $\begin{array}{l}\text { Ser. } \\
\text { Reg. B }\end{array}$ & Barbarus. & Synkrll. & $\begin{array}{l}X \rho o v . \\
\text { Xívt. }\end{array}$ & $\begin{array}{l}\text { richt } \\
\text { Zaihl }\end{array}$ & 1. Jahr. \\
\hline 1. Medon & 20 & 20 & 20 & 20 & 20 & 20 & 20 & $1068 / 7$ \\
\hline 2. Akastos & 36, & 36 & $36 f_{55}$ & 539 & 35 & $38 i_{55}$ & 36 & $1048 / 7$ \\
\hline 3. Archippos & $199^{55}$ & 19 & $19 l^{05}$ & $\begin{array}{ll}5 & 40[19]\end{array}$ & 19 & $17 l^{\mathrm{b}}$ & 19 & $1012 / 1$ \\
\hline 4. Thersippos & $\mathrm{s} 41$ & 41 & 41 & $-[41]$ & $40[41]$ & 41 & 41 & $993 /$ \\
\hline 5. Phorbas & 30 & 31 & 31 & 33 & 30 & 33 & 30 & $952 / 1$ \\
\hline 6. Megakles & $30 y_{58}$ & 30 & 30 & $28 j$ & $28 i_{56}$ & $30 \bigwedge_{56}$ & 30 & 922 \\
\hline 7. Diognetos & $281^{58}$ & 28 & 28 & $28 t^{5}$ & $281^{06}$ & $26\}^{00}$ & 28 & 892 \\
\hline 8. Pherekles & 19 & 19 & 19 & 15 & 19 & 19 & 19 & $864 / 3$ \\
\hline 9. Aripliron ${ }^{17}$ & ) 20 & 20 & 20 & 30 & 20 od. 31 & 33 & 20 & 845 \\
\hline 0. Thespiens & $7[27]$ & 27 & 27 & 40 & 27,40 & 40 & 27 & 825 \\
\hline 11. Agamestor & $\mathrm{r} 17[20]$ & 20 & 20 & 26 & 17,27 & 21 & 20 & 798 \\
\hline 12. Aischylos & $23^{L}$ & 23 & 23 & 23 & $14[23]$ & 23 & 23 & $778 / 7$ \\
\hline \multirow[t]{2}{*}{ 13. Alkmeon } & 2 & 2 & 2 & $10[2]$ & 2 & 2 & 2 & $755 / 4$ \\
\hline & 292531 & - & 316 & $332[34$ & $\begin{array}{r}299 \text { od. } 333 \\
{[343}\end{array}$ & 343 & 315 & \\
\hline
\end{tabular}

Die Einzelzahlen müssen als Summe 315 ergeben. Das thun sie auf den ersten Blick nirgends. Wenn aber der Kanon 316 Jahre aufweist, so sind das $315+1$, dem einen Jalır, das bei den Erechtheiden gestrichen ist und nun hier nachgeholt wird. Das scheint bei Phorbas geschehen zu sein, der im Text und bei Synkellos 30 Jahre hat, im Kanon 31. Die 34 im Barbarus und Xoovoyocepeiov sind Korruptel; ob aus $\vec{A}$ oder $\overline{A A}$ lässt sich nicht sagen; doch ist ersteres wahrscheinlich. Die Einzelzahlen des Textes liefern - die 27 für Thespieus statt 7 gleich eingesetzt - nur 312 Jahre. Der Barbarus hilft uns hier nicht viel, da er aus Africanus interpoliert ist²) und es schwer zu sagen ist, wie weit die Interpolation sich erstreckt. Auch weist seine Liste mehrfache Korruptelen und Verschiebungen auf. Es bleibt eigentlich nur Agamestors Zahl, der im Kanon 20, im Text 17 hat: $\left.{ }^{3}\right)$ Letzteres wird ein

1) Synkellos hat für die Könige Ariphron, Thespieus, Agamestor Varianten, deren

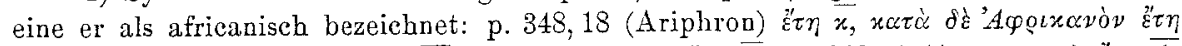

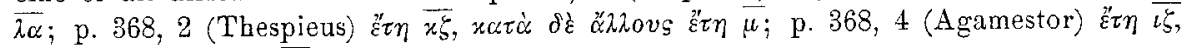
$x \alpha \tau \dot{\alpha} \delta \dot{\varepsilon} \alpha \dot{\alpha} \hat{\lambda}$ ov ${ }^{\prime \prime} \tau \eta \overline{\varkappa \zeta}$.

2) Das zeigen deutlich die Zahlen fưr Ariphron, Thespieus, Agamestor (s. Anm. 1). Es sind die z. T. Ieicht korrumpierten Varianten des Synkellos, die auch im Xoovoye.

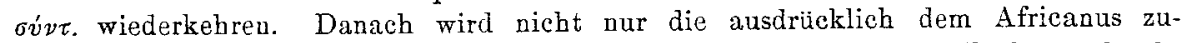
geschriebene erste Variante diesem gehören. Dass sonst die Liste des Barbarus durchaus die eusebische ist, wenn auch vielfach entstellt, bemerlat Scrwartz mit Recht.

3) Auch Schwartz setzt hier ein. Aber er muss eine niedrigere Zahl gewinnen, da er die Dauer der Dekaetie auf 80 Jahre erhöht hat. So schreibt er 10 statt 17. Die 20 Jahre des Kanons muss er danach für interpoliert erklärèn (Konigsisten S. 48). Aber ich verstehe nicht, wie er darin eine Stutze fur die Änderung der 17 in 10 sehen kann. Denn die Annabme einer solehen Interpolation von 10 Jahren in Kanon ist

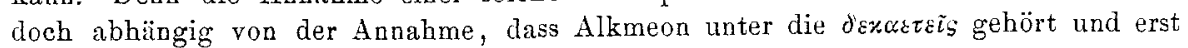


alter Schreibfehler sein, hervorgerufen durch die vorhergehende 27 ; denn die 17 Jahre kehren anch bei Synkellos wieder. Die 26 des Barbarus sind, wie Synkellos zeigt, Interpolation mit leichter Korruptel der Zahl. ${ }^{1}$ )

Damit sind wir mit Kastors Liste im reinen. Ich betone noch einmal, dass die erreichbare Sicherheit in den einzelnen Teilen der Liste verschieden ist. Für sicher halte ich die Ansätze der Könige von Kekrops bis Henestheus und die letzte Partie von Aischylos bis Kreon. Da haben wir den echten Kastor erreicht. In dem ganzen Nittelstïck aber, von Demophon bis Agamestor, beanspruchen die Ansätze nur einen Wahr scheinlichkeitswert, wenn sie sich auch von Kastors Daten keinesfalls weit - vielleicht gar nicht - entfernen. Aber einerseits das Eindringen der Interpolationen des Kanons in die Chronik und die Excerpta Barbari, andrerseits die Interpolation des Barbarus ans Africanus macht es unmöglich, aus der zerstörten Überlieferung das Original mit absoluter Sichel'heit wiederzugetwinnen.

\section{Die attische Königsliste des Pariers.}

Wir gehen zu der Königsliste der vom Parier benutzten Atthis über, um zu versuchen, wie weit sie sich wiederherstellen lässt. Dass diese Liste in Zahl und Folge der Könige die gleiche ist, wie die dem Kastor vorliegende, ist längst bemerkt worden. Auch die Differenz im Anfangsjahr bei gleichem Endjahr (683/2 Kreon) hat bereits Boвckн richtig erklärt. ${ }^{2}$ ) Kastor setzt Troias Fall - für ihn, wie für alle späteren Chronologen, unzweifelhaft das Fundamentaldatum der vorhistorischen Zeit - aufs Jahr 1184/3; der Parier giebt 1209/8 (1208/7). Zwischen beiden Ansätzen waltet eine Differenz von 25 Jahren ob.") Wenn wir

vom Interpolator aus ihnen vertrieben ist. Diese letztere Annabme aber bat ibn erst zu der Änderung von 17 in 10 gefiihrt. Das ist doch ein vollständiger Zirkelschluss, der nur deshalb nicht sofort auffält, weil seine Einzelglieder an weit von einander entfernten Stellen von ScHwartz' Abbandlung stehen.

1) Die Gesantsumme der lebenslänglichen Archonten ist bei Synkellos (mit den Varianten) und im Xoovoyecosĩov die gleiche (343), um 28 Jahre böhere als bei Eusebios. Der Barbarus hat noch $1 \mathrm{Jahr}$ unehr. Alle drei bilden einen Zweig der Überlieferung, der sich durch Interpolationen aus Africanus charakterisiert. Die Versebiedenheiten in den Einzelzahlen werden ausgeglichen. So giebt der Barbarus Akastos 39, Pherekles 15 Jahre; Synkellos 35 und 19. Phorbas hat beim Barbarus 33, bei Synkellos 30. Dafür giebt er Ariphron 30 statt 31 , Agamestor 26 statt 27 . So bleibt ihm noch 1 Jahr mehr. Das Xeovoye. hat in der Gruppe Akastos-Archippos $1 \mathrm{Jahr}$ mehr als Syukellos, fuir Phorbas 3, fuir Ariphron 2 mehr; in summa 6. Dafur erhält Agamestor nur 21 statt 27.

2) In dem chronologischen Kanon, den er seiner Ausgabe des Marmors beigegeben hat. Im ubrigen ist er aber von der richtigen Ansicht über die attische Liste KastorEusebs weit entfernt. Die Einsicht in ihre Überlieferung wird erst Schwarrz verdankt.

3) Die Existenz dieser Differenz scheint der kunftige Herausgeber des Marmors Munro, Classical Review XV (1901) S. 154 leugnen zu wollen; offen gesagt, verstehe ich allerdings seinen Gedankengang nicht recht: ${ }_{\pi}$ It is worth noting that the two slips, 
sehen, dass die gleiche Differenz zwischen den Anfangsjahren beider Listen (Beginn des Kekrops 1581/0 im Marmor, 1556.5 bei Kastor) und weiterhin vorliegt, so ist es allerdings klar, dass die Daten der Atthis umgerechnet sind auf die troische dera von 118t/3. Diese Umrechnung hat aber schwerlich erst Kastor vorgenommen; sie war Sache dessen, der als erster das troische Datum der Atthis um 25 Jahre herabschob, dabei aber die Gleichung zwischen Troias Fall und Menestheus' letztem Jahre wahren musste. ${ }^{1}$ ) Das kann nur Eratosthenes selbst gewesen sein. Denn er hat das später fast allein herrschend gewordene Datum für Troias Fall 1184/3 unter Benutzung der spartanischen Königsfasten berechnet, ${ }^{2}$ ) und es ist wohl unzweifelhaft, dass er in seinen Xoovorocpia neben der spartanischen anch die attische Liste, nach seiner neuen Aera umgerechnet, anfgestellt hat. Damit rückt der Ursprung auch der kastorischen Liste in das 3. Jahrhundert v. Chr. hinauf. Für uns aber entsteht die Frage: wo hat Eratosthenes die durch Herunterschieben des troischen Datums nötig gewordenen Abstriche von 25 Jahren vorgenommen?

Es ist bedanerlich, dass wir die Liste der vom Parier benutzten Atthis nicht vollständig besitzen, sondern sie erst aus den einzelnen Erwähnungen der eponymen Könige rekonstruieren müssen. Aber glücklich

Medon 13 for Medon 19, and Menestheus 2 for Menestheus 22, would, if taken seriously, throw the historical reckoning 26 years back on the numeral, and that is precisely the intervall, by which the Marble differs from the Canon of Eusebius. Possibly it may not after all be the engraver who is to blame." Munko wird doch nicht im Ernst verlangen, dass wir diese beiden Versehen des Steinmetzen seriously nehmen. Die

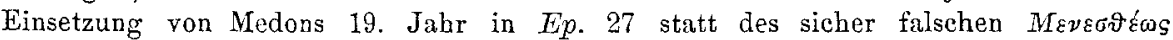

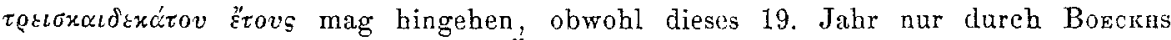
falscbe Behandlung der eusebischen Überlieferung zustande gekommen ist. Aber das 2. Jahr des Menestheus für Troias Fall ist eine bare Unmöglichkeit, wenn der Auszug der Griechen in sein 13. Jahr gesetzt wird (s. oben S. 428, 2). Und was machen wir mit all den Epochenzahlen des Marmors? Aber es ist wirklich unnutz, hier zu polemisieren. - Dass diese Differenz auch in verschiedenen Einzeldaten obwaltet, sah schon Bовскн. In anderen führt sie unter Beibehaltung der Abstände zwischen dem betreffenden Ereignis und Kreons Arehontat zur Verschiebung in eine andere Regierung. Um nur ein Beispiel zu nennen. Das Marmor setzt Ep. 6 Hellen und die Entstehung. des. Hellenennamens 1257 Jahre vor Diognetos, also a. Chr. 1520/19, unter Amphiktyon. Eusebs Kanon hat dasselbe s. a. Abr. $498=$ a. Chr. 1519/8, aber natürlich unter Kekrops.

1) Die ganze Operation des Eratosthenes ist ein Beweis, wie fest diese Gleichung in der Überlieferung war; viel fester als der Ansatz der ionischen Wanderung in Medons Regierung. Die hat Eratosthenes, da er das Intervall zwischen Troias Fall und Heraklidenrückkehr statt auf 60 (66) auf 80 Jahre angab, wie 'Thukydides und wohl schon friihere Autoren, einfach aus Medons in Akastos' Regierung geschoben (s. unten S. 434). Hütte er mit dem Synchronismus źwischen Troias Fall und Menestheus ebenso umspringen können, so hätte er sich die ganze Umrechnung einfach sparen können.

2) Clemens Alex. Strom. I 138 p. 402 P. Vgl. Philol. Unters. XVI S. 39 f. 
fügt es sich, dass uns der Chronist für den ersten wichtigsten Teil der Liste von Kekrops bis Troias Fall nicht nur genïgende Fixpunkte giebt, sondern auch dass diese gut erhalten sind. Zu berücksichtigen ist aber immer, dass wir bei jedem dieser Fixpunkte die Wahl zwischen zwei Jahren haben, solange nicht andere Zeugnisse die Frage entscheiden. Der Chronist giebt uns nun folgende wichtige Daten:

bei Kastor.

1. Kekrops 1. Jahr: 1582/1 oder $1581 / 0$ (Epoche 1) $1556 / 5$

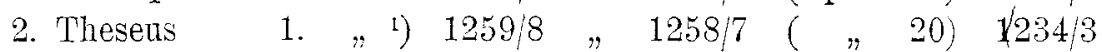

3. Menestheus' 1. " $1230 / 29 " 1229 / 8$ ( " $" 23)$ [1205/4]

4. Troias Fall "1209/8 " $1208 / 7\left(\begin{array}{lllll} & \text { " } & 24) & 1184 / 3\end{array}\right.$

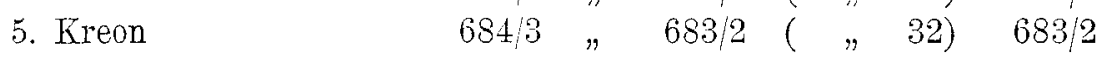

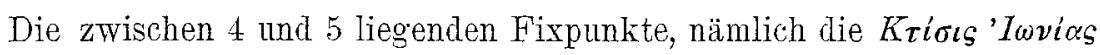
(Ep. 27) und das 21. Jahr des Aischylos (Ep. 31), sind, jener durch

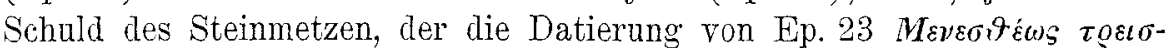

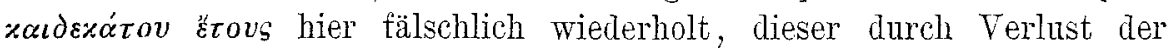
Epochenzahl für uns nicht mehr ohne weiteres festzustellen. Sie werden unten besprochen werden. - Vergleichen wir diese Angaben mit den entsprechenden Daten Kastors, so ergiebt sich neben der Beobachtung, dass die Differenz vom Anfang der Liste bis Troias Fall die gleiche bleibt, als wichtigste Thatsache, dass der Chronist den Fall Troias ins 373. oder 374. attische Königsjahr setzt. Wir haben davon oben (S. 422 f.) bereits Nutzen gezogen und konstatiert, dass der Antor von Kastors Liste, d. h. Eratosthenes, dieses Intervall ohne Änderung ans der Atthis übernommen hat. Dieser Umstand aber erlaubt eine Entscheidung über die Zählweise des Chronisten. Denn da Kastor Troias Fall mit dem 373. attischen Königsjahre glich (1556/5-1184/3), so dürfen wir das gleiche für den Parier annehmen und haben damit die Möglichkeit, die Doppeldaten auszuschliessen. Der Chronist hat Ep. 1 von $263 / 2$ gerechnet, wie wir das oben (S. 413f.) bereits für Kreons Jahr (Ep. 32) festgestellt haben, Ep. 23. 24 dagegen von 264/3. Wir gewinnen also:

1) Dass hier das 1. Jahr des Theseus anzunehmen ist, zeigt die Fassung der

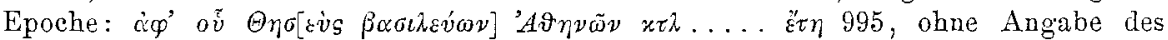
Eponymos, analog der Epoche 1, dem 1. Jahre des Kekrops: ‘் ' o $\dot{\varepsilon} \beta c \sigma i \lambda \varepsilon v \sigma \varepsilon \ldots . . . \varepsilon^{\prime \prime} t \eta ~ 1318$, ebenfalls ohne Eponymos. Schon dadurch erledigt sich m. E. die Behauptung von Schwartz, Königslisten S. 55 f, das 1. Jahr des Thesens sei in 1279/8 zu verlegen auf Grund des Minosansatzes im Marmor Ep. 19 ins Jahr 1295/4, der Überlieferung (Plutarch, Thesens 15, 17; Biblioth. epit. I 7), dass Theseus beim dritten oktaeterischen $\delta \alpha \sigma \mu \delta$ s mit nach Kreta gegangen sei und der Gleichsetzung von 'Theseus' Ruckkehr und Aigens' Tode. Es fuhrt zu weit, hier zu erwägen, wie die scheinbare Diskrepanz des Pariers gegen die gewöhnliche Überlieferung zu erklären ist. Es giebt mehrere Möglichkeiten. Aber sieher ist, was der Chronist selbst giebt, Theseus' Beginn.

Beitrige z. alten Gesehichte II 3. 
Kekrops 1. Jahr

$1581 / 0$

Nenesthelis' 1. Jahr

$1230 / 29$

Troias Fall $=$ Menestheus' 22. Jahr $=373$. der Liste 1209/8

Da nun das Intervall zwischen Kekrops und Troias Fall im Marmor und bei Kastor das gleiche ist, da beide ferner in Zahl und Folge der vortroianischen Könige übereinstimmen - Kekrops II, aus dessen Regierung der Pariel nichts notiert, wird als Glied seiner Liste erwiesen

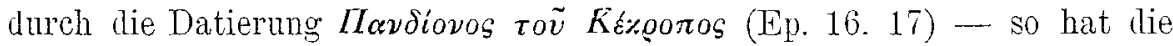
Annahme, dass auch die Regierungszahlen dieser 11 ersten Könige im Narmor die gleichen waren, wie bei Kastor, zunächst alle Wahrscheinlichkeit für sich. ${ }^{1}$ ) Sahen wir ja doch, dass auch das Intervall von 25 Tahren, soweit wir urteilen können, konstant bleibt. Stellen wir danach die Liste auf:

$\begin{array}{lrrrrr}\text { 1. Kekrops } & \text { regiert } & 50 & \text { Jahre von } & 1581 / 0 \\ \text { 2. Krana0s } & . & 9 & " & " & 1531 / 0 \\ \text { 3. Amphiktyon } & " & 10 & " & " & 1522 / 1 \\ \text { 4. Erichthonios } & " & 50 & " & " & 1512 / 1 \\ \text { 5. Pandion } & " & 40 & . & " & 1462 / 1 \\ \text { 6. Erechtheus } & " & 50 & " & " & 1422 / 1 \\ \text { 7. Kekrops II } & " & 40 & " & " & 1372 / 1 \\ \text { 8. Pandion II } & " & 25 & " & " & 1332 / 1 \\ \text { 9. Aigeus } & " & 48 & " & " & 1307 / 6 \\ \text { 10. Thesells } & . & 29 & . & " & 1259 / 8 \\ \text { 11. Wenestheus } & . & 22 & " & " & 1230 / 29-1209 / 8\end{array}$

Sehen wir uns nun die einzelnen Datierungen des Marmors an, so erscheinen folgende Eponymen für folgende Jahre (von 263/2 ge(rechnet). $\left.{ }^{2}\right)$

\begin{tabular}{|c|c|}
\hline 1. Kekrops & Ep. 1 für $1581 / 0$ \\
\hline & $" 2,1573 / 2$ \\
\hline 2. Kranaos & $1531 / 0$ \\
\hline$"$ & $" 4 . .1528 / 7$ \\
\hline
\end{tabular}

1) Von vornherein war die Annahme nicht etwa selbstverständlich. Wir haben eine - allerdings nur eine abweichende Angabe. Amphiktyon regiert nach [Apollodor.] Bibliot\%. III 187 nicht 10 Jahre, wie bei Kastor, sondern 12. Die Zahl ist merkwürdig. Denn wenn wir sie in die oben herzustellende Liste des Pariers einfuhren, so kommt Kekrops' 1. Jahr auf $1583 / 2$, d. h. 900 Jahre vor Kreon, ein Intervall, das jedenfalls ursprünglich auch in der Atthis des Pariers vorlag., Offenbar hat er absichtlich diese runde Zahl gekurzt (s. oben S. 413, 2).

2) Fragezeichen sind gesetzt, wo I ticken des Steines die Feststellung des Datums unmöglich oder unsicher machen. 
3. Amphiktyon Ep. 5 für $1521 / 0(1522 / 1)$

$\begin{array}{lllll}" & " & 6 & & 1520 / 19 \\ " & " & 7 & & 1518 / 7 \\ " & & 8 & . & 1515 / 4\end{array}$

4. Erichthonios ", 9?", $1510 / 9$

$" \quad$ "10 , 1505/4

5. Pandion " $11, \quad$ ?

6. Erechtheus , $12, " 1409 / 8^{1}$ )

"1:, "1408/7

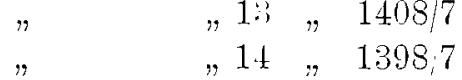

7. Kekrops

$" 15 "$ ?

8. Pandion II $\quad " 16 \quad, 1325 / 4(?)$

$\begin{array}{ccc}\text { 9. Aigeus } & " 17 & \\ & , 18 & \text { ? }\end{array}$

$, \quad " 19,1294 / 8$

10. Theseus " $20,1259 / 8$

" $\quad 21 \quad$., $1256 / 5(1555 / 4)$

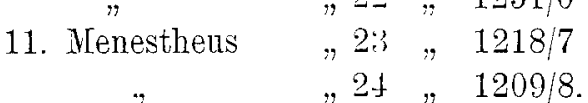

Keines dieser Daten widerspricht der aufgestellten Tabelle. Das ist immerhin ein unverächtlicher Beweis für ihre Richtigkeit. Denn aus der nur 10 jährigen Regierung des Amphiktyon giebt uns der Chronist 4 Daten. Aber noch mehr spricht eine andere 'Thatsache für die Richtigkeit der Tabelle. Gurscumin besonders hat es als Eigentümlichkeit der antiken Chronologen erkannt, dass sie ein durch Königsnamen datiertes Ereignis mit Vorliebe in das erste Regierungsjahr des betreffenden Herrschers setzen. ${ }^{2}$ ) Nun ist aber in Ep. 3 das Datum gleich dem 1. Jahre des Kranaos in unserer 'labelle; auch Ep. 5 führt (von 264/3 gerechnet) in das erste .Jahr des Amphiktyon; Ep. 1t ist in die Mitte von Erechthens' Regierung gesetzt. Ep. 1 und 20 stehen auf dem 1. Jahre des Kekrops und Thesens. Alles dies bildet wohl einen sicheren Beweis dafür, dass Fastors Liste bis zum Falle Troias nicht nur in Zahl und Folge der Könige, sondern auch in den Dauern der Einzelregierungen mit der des Yarmors stimmte. Wir haben also Recht daran gethan, die durch den Interpolator des Eusebischen Kanons entstandene Trübung von Kastors Liste durch die Zahlen des Marmors fortzuschaffen. ${ }^{3}$ )

1) Überliefert ist in beiden Epochen 1145. Muxro korrigiert wohl richtig die erste Zahl in 1146.

2) eine ähnliche Rolle spielen, wenn das erste Jahr aus irgend einem Grunde ausgeschlossen war, das mittlere und las letzte.

3) S. oben S. $428 \mathrm{f}$. 
Aus dem Umstande, dass die Differenz zwischen den Daten Kastors und des Marmors ihren Ursprung in der verschiedenen Ansetzung von Troias Fall hat, ergiebt sich, dass Eratosthenes die notwendigen $A b-$ striche erst bei den nachtroianischen Herrschern vorgenommen hat. Die Frage, wo das geschehen ist, ist selir schwierig, da uns der Chronist für die Zeit von Troias Fall bis Kreon nur eine sehr geringe Zahl von Daten zur Vergleichung liefert, und diese zum Überfluss noch recht mangelhaft erhalten sind. Dennoch, glaube ich, kommen wir zu einem Resultat, vielleicht dem wichtig'sten unserer ganzen Untersuchung.

Demophon ist nach Ep. 25 Eponymos für 1202/1 (1201/0). Das ist sein 7. Jahr; er beginnt 1208/7, nach Kastor 1183/2. Die Differenz

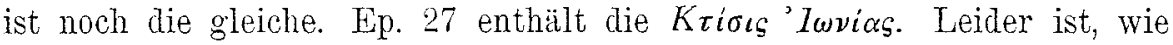

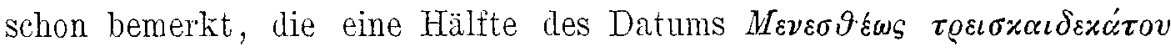
žzovs aus Ep. 23 wiederholt durch Versehen des Steinmetzen. Zwar dass Mkdovros einzusetzen ist, leidet wohl kaum einen Zweifel. Denn eben der gleiche Anfang der beiden Namen hat hier, wie in dem analogen Falle von Ep. 6,1) das Auge des Steinmetzen abirren lassen. Aber das Jahr ist unrettbar verloren.") Auch die Epochenzahl ist nicht vollständig erhalten; doch erlaubt der Rest .... AIII nur die Ergänzung 823 oder 813, führt also auf 1087/6 (1086/5) oder 1077/6 (1076/5). Der Abstand von Troias Fall ist also 122 (123) oder 132 (133) Jahre; beide Zahlen

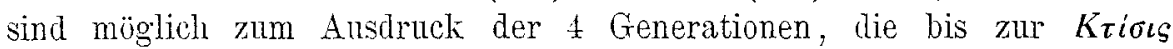
'Iwvias verfliessen. ${ }^{3}$ ) Die Differenz zwischen den beiden Daten für die ionische Wanderung beträgt also — für das Marmor 1087/6 vorausgesetzt, 43 Jahre d. h. $25+18 .{ }^{4}$ ) Denu Kastor hat mit Eratosthenes zwischen Troias Fall und 'Iwvias Kriбıs das erhöhte Mischintervall von $80+60=$ 140 Jahren gelegt, während die Atthis 4 gleichartige $\gamma \varepsilon v \varepsilon \alpha i$ rechnete.

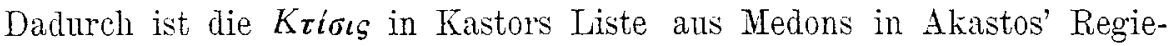
rung verschoben. Die Differenz aber gegen den Parier ist noch die gleiche. - Ep. 28 fehlt der Eponymos. - Ep. 29 ist Diognetos Eponym fuir $907 / 6(906 / 5)$. Nach Kastor regiert er 892/1-865/4. Da der Parier

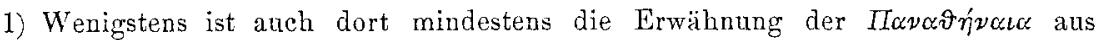

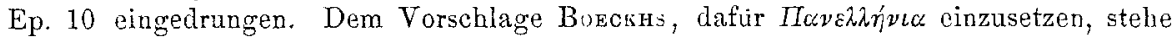
ich allerdings sehr skeptisch gegenuber.

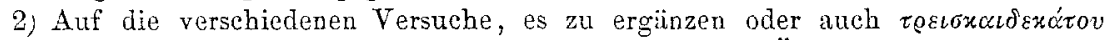
žcovs zu retten, brauche ich nicht einzugehen. Sollte die Übereinstimmung in der Dauer der Einzelregierungen zwischen Kastor und dem Parier sich noch tiber Troias Fall hinaus fortsetzen, so wirden uns 122 Jabre in Medons 7., 132 in Medons 17. Jahr fuhren. Aber die Voraussetzung ist unsicher. Vgl. aber auch S. 438, 1.

3) Fuir die Ergänzung 813 - also 132 uach Troias Fall - entscheiden sich Вовски und Schwartz, Konigslisten S. 55; fur 823-122 nach Troia - Gurschmo und E. Meqer, Forschungen I S. 172, 3.

4) Bei dem Ansatz der Kíiols auf $1077 / 6$ betriigt die Differenz 25+8. Das Resultat bleibt das gleiche. 
Diognetos' Jahr nicht angiebt, lässt sich nicht sagen, ob die Differenz noch in vollem Umfang besteht. Ausgeglichen ist sie jedenfalls noch nicht. - Erst die beiden letzten Eyochen der Königszeit, Ep. 30. 31 geben uns die gewünschte Aufklärung. Zwar ist von dem Eponymen der Ep. 30, Jahr 895/4 (89t/3) nur die Endung - $\iota_{0}$ uv $^{1}$ ) erhalten. Aber

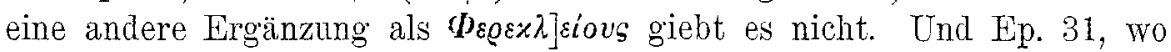

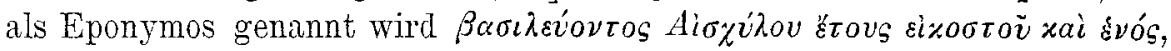
fehlt zwar die Epochenzahl, aber zwei andere Zeitbestimmungen helfen uns aus der Verlegenheit. Fp. 30 nämlich wird Pheidon, der Tyrann

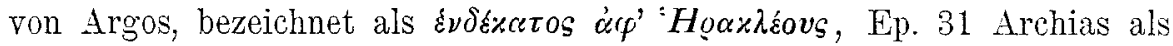

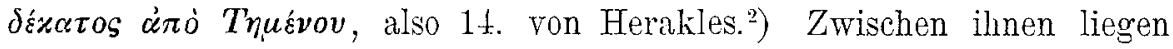

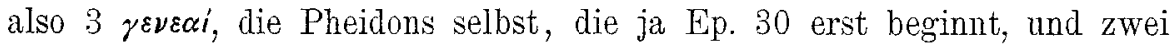
weitere. Pherekles regiert nach Kastor 864/3-846/5 und Aischylos' 21. Jahr ist nach demselben gleich 758/7. Zwischen beiden liegen also

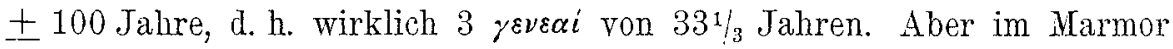
scheint die Sache anders zu liegen. Pherekles ist hier Eponym für 894/3 (895/4). Selbst wenn wir annehmen, dass Ep. 30 unter sein 1. Jalrr gestellt ist, wie ja liäufig im Marmor, so liegt sein Anfang doch 30 Jahre früher als in Kastors Liste. Die Differenz zwischen des Pariers und und Kastors Daten hat sich mit der Annäherung an den ersten festen Punkt, den Grundstein des ganzen chronologischen Gebäudes, Kreons Archontat, nicht wie man denken sollte, verringert; sie ist vielmelhr ge-

1) So nach den neuen Vergleicbungen. Die Orthographie wie im Fragment B Ep. 24 u. s.

2) Lch kann die Pheidonfrage hier naturlich nicht aufrollen; für unseren $Z$ weck genügt es zu konstatieren, dass diese beiden Bestimmungen der $\gamma \varepsilon \nu \varepsilon \alpha i$ die Änderung Gutscamids in der Epochenzahl von Ep. 30, durch die er Pheidon 100 Jahre tiefer riicken will, unmöglich machen. (Gurschmid, $K l$. Schr. IV 77, 2; ihm stimmt zu Dopp, Quaest. d. Marm. Pario S. 25.) Die Verwunderung Boeckns "aetates hinc undecim sunt sane largints computatae" - weil nämlich Herakles im Marmor zuerst Ep. 18 zwischen 1307/6 und 1295/4 genannt wird, zwischen ihm und Pheidon also 400-412

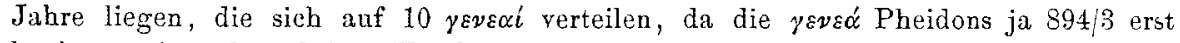
beginnt - ist unberechtigt. Herakles, der Zeitgenosse des 'Theseus und der Argonauten, gehört regulär in die $\gamma \varepsilon \nu \varepsilon \dot{\alpha}$ vor Troias Fall. Wenn ihn der Chronist \pm 100 Jahre vor

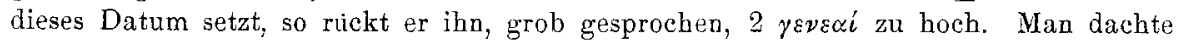
sich eben die $\gamma \varepsilon v \varepsilon \dot{\alpha}$ des Herakles selbst wegen der verschiedenen ihn betreffendeu Synchronismen ausgedehnter oder besser, Herakles' Leben erstreckt sich über mehrere $\gamma \varepsilon v \varepsilon c i^{\prime}$. Rechnen wir von Troias Fall, 1209/8, von dem Pheidon der 10. ist, so stimmen

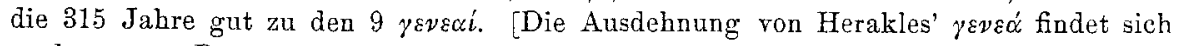
auch sonst. Ps. Apollodor. fr. 119. Philol. Unters. XVI 402 rechnet ca. 100 Jahre

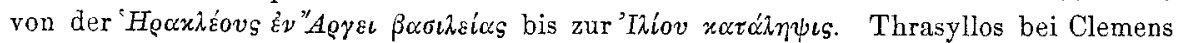
Alex. Strom. I 136. 137 setzt die Argonauten 90, die Olympienstiftung des Herakles 45 Jahre vor Troias Fall. Auch Euseb, Chron. I 186, 10 notiert wie der Parier die

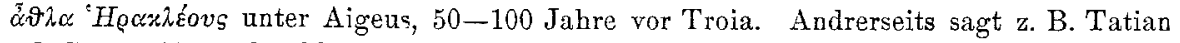

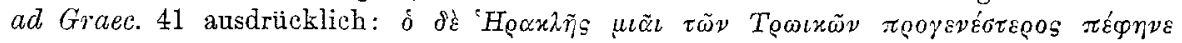

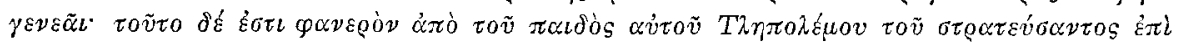
"Ihıov. Und das ist die ubliche Rechnung.] 
wachsen. Eratosthenes muss also irgendwo zwischen Demophon und Plierekles eine der Regierungen um mindestens 5 Jalire verlängert haben. ${ }^{2}$ ) Ich weiss nicht, ob schon jemand auf diese merkwürdige Thatsache aufmerksam gemacht hat. Andrerseits hat man sich gewöhnt, Aischylos' 21. Jaln als fest zu betrachten. Sowohl Boecke wie Gurscmmid -ergänzen die Epochenzalil von Ep. 31 unbedenklich so, dass das kastorische Jahr (oder was sie dafür halten) 758,7 herauskommt. Zwischen $894 / 3$ und $758 / 7$ aber liegen 136 (137) Jahre, d. l. 4 yeveci. Die Annahme, der Parier - oder auch seine Quelle - habe hier plötzlich Generationen zu 45 statt zu 33 Jahren gerechnet, ist natürlich ausgeschlossen. Oder liat der Clironist Ep. 30 die Generationen mit Linschluss beider Termini gezählt, Ep. 31 dagegen Temenos ausgeschlossen, sodass er nach der üblichen Zählweise Archias nicht den 10., sondern den 11. von Temenos hätte nennen müssen? Unmöglich wäre das nicht; aber es ist nicht nur wegen der Inkonsequenz bei zwei oftenbar mit einander in Zusammenhang stehenden Daten, die sicher derselben Quelle entnommen sind, einem Autor, der nach Generationen rechnete,,$^{2}$ ) im höchsten Grade unwahrscheinlich. Eher müssen wir uns doch fragen, welches Recht wir eigentlich zu der ohne Beweis hingestellten Annahme liaben, das 21. Jaln des Aischylos liabe bei Kastor und beim Parier dem gleichen Jahre vor Christus oder attisch vor Kreon entsprochen. ${ }^{8}$ ) Die Frage stellen, heisst die Annahme verwerfen. Denn wenn wir für Diognetos den Fortbestand der Differenz in den Daten konstatieren können, wenn wir sie für Pherekles noch angewachsen finden, und wenn schliesslich nach dem Parier zwischen Pherekles und Aischylos' 21. Jahr

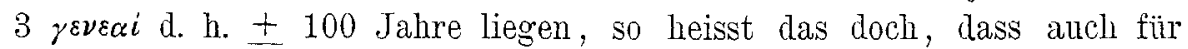

1) Wo das geschehen sein kam, werden wir später sehen.

2) Wer das war, kann bier aicht untersucht werden. Die Quellenfrage des Marmors muss im Zusammenhange behandelt werden.

3) Ich möcbte, obwohl das eigentlich unnötig ist, doch ausdrucklich darauf hinweisen, dass uns die Varianten des Africanus, die gerade fur Ariphron Thespieus Agamestor erbalten sind (s. oben S. 428, 1) und die eine um 31 Jahre böhere Gesamtsumme ergeben als die entsprechenden Zablen Kastors, hier garnichts helfen, so sehr Art und Stellung dieser Varianten die Annahme zu begunstigen scheinen, dass Africanus bier die Zahlen der Atthis aufweist, die Kastor gekurzt hat. Aber SchwarT\% a. a. O. bemerkt mit Recht, dass, da Africanus das gleiche oder doch nur um 1 Jahr verschobene troische Datum hatte wie Euseb, alle Verschiedenheiten zwischen seiner und der Kastor-Eusebischen Liste unmittelbar in denselben Teilen der Liste auch wieder ausgeglichen sein müssen; d. h. die 51 Jahre, um die Kekrops bei Africanus früher beginnt als bei Euseb (s. S. 418, 1), muissen bei Menestheus' letztem Jabre wieder eingebracht sein, denn Troias Fall ist bei Euseb 1184/3, bei Africanus 1183/2; ebenso mussen die in der Reihe Ariphron-Agamestor überśchiessenden 31 Jahre bei anderen

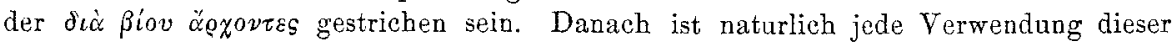
Varianten zur Ausgleichung der zwischen der Atthis des Marmors und Eratosthenes bestebenden Diskrepanzen ausgeschlossen. 
Aischylos' Ansatz die Differenz noch bestand. Also hat der Verfasser von Kastors Liste, Eratosthenes, seine Abstriche von nun mindestens 30 Jahren an den Regierungen zwischen Aischylos und Kreon vorgenommen.

Dies Resultat ist auf den ersten Blick überraschend genug; denn bisher hatte man diesen letzten Teil der attischen Liste als chronologisch und historisch wenigstens leidlich sicher betrachtet. Eben diese stillschweigende Annahme hat ja zu der den eigenen Angaben des Pariers durchans widersprechenden Finführung von Kastors Ansatz für Aischylos in die Chronologie des Narmors gefülnt. Nach unserem Resultate, dass Eratosthenes seine Abstriche friihestens bei Alkmeon vorgenommen hat, bieten sich uns drei Möglichkeiten für die Einzelheiten seines Verfahrens. Wer die Zahll der $\delta \varepsilon x \alpha \varepsilon \tau \varepsilon \tilde{\iota}$ für überliefert hält, der muss sich den Ab-

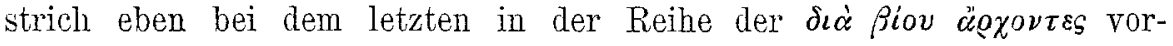
genommen denken, bei Alkmeon, dessen Regierung nach Kastor nur 2 Jahre dauert. Diese auffällige Kürze hat in Verbindung mit der That-

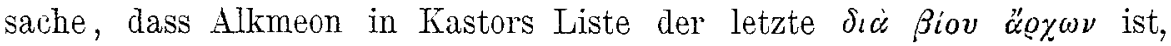
manche V ermutungen der Gelehrten über seinen gewaltsamen Sturz und eine damit verbundene Verfassungsänderung gezeitigt. Die Berechtigung dieser Vermutungen zu prïfen, ist hier noch nicht der Ort. Wer aber der kastorischen Überlieferung über die $\delta \varepsilon x \alpha \varepsilon \tau \varepsilon \check{\iota s}_{S}$ weniger glïubig gegeniibersteht, der wird vermuten, dass Eratosthenes seine Änderungen eben bei den $\delta \varepsilon x \alpha \varepsilon \tau \varepsilon i \bar{s}$ vorgenommen hat. Dass eine solche Vermutung von vornherein nicht der Wahrscheinlichkeit entbehrt, wird jeder zugeben, (ler sich der Rolle exinnert, die derartige Reihen von befristeten Beamten zwischen dem letzten Könige und dem ersten historisch beglaubigten . Tahre in den verschiedenen chronographischen Systemen spielen. Ich

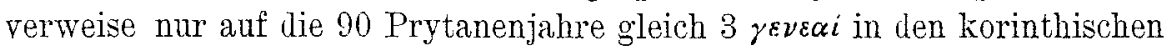
Fasten zwischen dem letzten Herrscher aus dem Bakchiadenhause und dem Beginne des Kypselos. Wie weit wir eine gleiche Chronologenmache auch für die attische Liste und speziell für die $\delta \varepsilon x a \varepsilon \tau \varepsilon \bar{u}$ annehmen dürfen, wird die historische Betrachtung lehren. Vorläıfig muss es uns genügen die Möglichkeit zu konstatieren, dass die Differenzen zwischen der Liste des Pariers und der kastorischen erst in dem letzten Teile der Fasten ihre Ausgleichung fanden. Die Möglichkeit der Ausgleichung ist wieder eine doppelte. War 894/3 nach dem Parier wirklich das 1. Jahr des Pherekles, so könnte die gerade 30 jährige Differenz $(894 / 3: 864 / 3)$ uns veranlassen, der Atthis des Marmors statt der 7 kastorischen $\delta \varepsilon x \alpha \varepsilon \tau \varepsilon i \varsigma$ deren 10 zuzuschreiben. 100 Jahre der Dekaetie nach Abschaffung des Königtums, 3 Generationen, ganz analog den 3 Generationen der korinthischen Prytanen oder auch der einen der Karneenpriester in der sikyonischen Liste. Wir würden uns also den letzten Teil der ursprünglichen Liste so zu denken haben: 894/3 Pherekles' 1. Jahr. 784/3 Alkmeons 


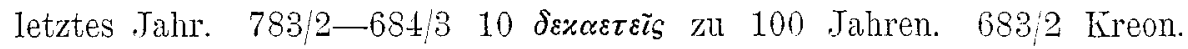
Die 5 Jahre, um die Kastor, wie wir oben sahen, dann in nachtroianischer Zeit und vor Pherekles eine der Regierungen verlängert haben muss, hat er vielleicht zwischen Demophon und Melanthos hinzugefügt, um für des letzteren Beginn das charakteristische Jahr 1126/5 (430 Jahre nach Kekrops $=11+2$ Generationen) zu gewinnen. ${ }^{3}$ ) Aber die Annahme, dass Ep. 30 unter Pherekles' 1. Jahr gestellt sei, ist eben nur eine Annahme, wenn auch auf den ersten Blick wenigstens eine lockende. Ob aber dem Eratosthenes eine so gewaltsame Massregel, wie die Streichung von drei Namen aus der Liste, zuzutranen ist ${ }^{2}$ ) und ob uns die Überlieferung - es könnte sich nur darum handeln, dass Alkmeon als letzter König so fest bezeugt war, dass Eratosthenes damit rechnen musste - ein Recht zu dieser Annahme giebt, das wird wieder erst später zu untersuchen sein.

So bleibt uns noch die dritte Möglichkeit. Eratosthenes kann die

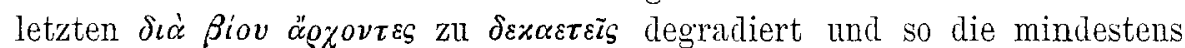
30 jührige Differenz wieder eingebracht haben. Die Überlieferung giebt uns einen Anhalt, wo das geschehen sein könnte. Denn sie bezeichnet Hippomenes als den letzten König ans dem Kodridengeschlecht. Sollten etwa die ersten vier $\delta \varepsilon x a \varepsilon \tau \varepsilon \check{c}_{\zeta}$ Kastors, Charops Aisimides Kleidikos Hip-

1) Wir bekämen da sehr merkwürdige Zablen. Die Könige Demophon, Oxyntes, Apheidas, Thymoites regieren nach Kastor 57 Jabre (s. oben S. 427). In der Liste der Atthis hätten sie 52 Jahre regiert. Es wurden sich folgende Zablen ergeben:

Troias Fall

Demophons 1. Jabr

Melanthos' 1. Jahr

Medons 1. Jahr

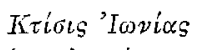

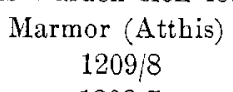

$$
1087 / 6(1086 / 5)
$$

Kastor

$1184 / 3$

$1183 / 2$

(57)

(58) $1126 / 5$

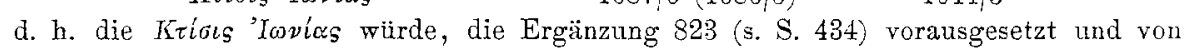
263/2 gerechuet, wirklich in Medons 13. Jahr fallen, der Fehler des Steinmetzen

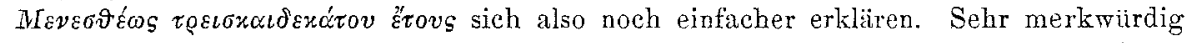
wäre auch das so sich ergebende 1. Jahr des Melanthos im Marmor, 1156/5, 400 Jahre nach Kekrops' Beginn in Eratosthenes' Liste. Sollte Eratosthenes hiervon ausgegangen sein, also von dem Jahre, in dem die Erechtheiden abgelöst werden durch die Melanthiden, und einfach 12 Generationen $=400$ Jahren zuruckgerechnet haben? Aber wie gesagt, die Voraussetzungen der Rechnung sind zu unsicher.

2) Freilich würden uns die spartanischen Fasten des Eratosthenes die Analogie zu einem derartigen Verfahren bieten. In dem Stemma der Eurypontiden hat er Polydektes, bei Herodot VIII 131 Vorg̈̈nger, bei Pausanias III 7, 2 Nachfolger des Eunomos, einfach gestrichen. S. Philol. Untersuch. XVI S. 89. 110. Dagegen hat er in das Agiadenstemma einen König Menelaos eingefuhrt, den weder Herodot noch Sosibios kannten. So wird man ein gleiches Vorgehen in der Gestaltung der attischen Liste nicht a limine ablehnen können. 
pomenes, die noch aus dem alten Herrscherlause waren, in der Liste,

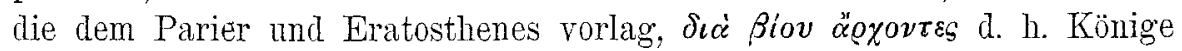
gewesen sein? Die Verfassungsänderung, die das Geschlechtskönigtum abschrafite, hätte dann gleichzeitig in einer Befristung des Amtes auf 10 Jahre bestanden. Es ist sehr zu bedauern, dass der Parier mit einer Unterlassungssünde - vielleicht der schwersten unter den vielen, die er begangen hat - aus der Zeit der $\delta \varepsilon z \alpha \varepsilon r \varepsilon i \bar{s}$ kein Ereignis notiert. So wissen wir nicht, ob und wieweit ilm diese Beamten noch als "Könige" galten oder ob er sie als üoxov $\tau \varepsilon \varsigma$ bezeichnet hat. Die Daner der Dekaetie bis zur Befristung des liüchsten Amtes auf 1 Jahr hätte bei der Annahme unserer dritten Möglichkeit 30 Jahre gewährt, d. h. eine Generation. Anch das würde wieder chronographische Nache verraten.

So hat uns die bis zu Ende durchgeführte rein chronographische Vergleichung der beiden uns erhaltenen Rezensionen der attischen König'sliste zu dem Resultat gefülırt, dass die zwischen ihnen bestehenden Differenzen im Ansatz der Einzelregierungen erst in dem letzten, der historisch sicheren Reihe der Jaluresbeamten unmittelbar vorhergehenden Teile ansgeglichen sind; dass also die attischen Fasten im 3. vorchristl. Jahrhdt. selbst in ihrem letzten Teile nicht historisch feste Daten aufwiesen, sondern Änderungen einschneidender Art erlaubten auf Grund des doch erst sekundären Datums von Troias Fall. Dieser Thatbestand erlaubt uns sichere Schlüsse auf Wert und Umfang der Überlieferung, die in dem chronographischen System der Atthis wie des Eratosthenes steckt. Er zeigt vor allem, dass der chronographische Wert der Fasten gleich Null ist. Die Ausgleichung selbst kann, wie wir sahen, auf drei verschiedenen Wegen erfolgt sein. Eine Entscheidung zwischen ihnen zu treffen, ist auf Grund der hier vorgelegten chronographischen Untersuchung nicht möglich. Denn chronographische Wahrscheinlichkeitsgründe lassen sich für jede der drei Möglichkeiten ins Feld führen. Hier also hat die historische Untersuchung einzusetzen. Unter Benutzung der auf chronographischem Wege gewonnenen Resultate und mit Heranziehung der anderweit vorhandenen Überlieferung muss der Versuch gemacht werden, die Entwicklung des attischen Königtums zu erkennen. Wir werden vor allem zu fragen haben, wieviel historischen Wert die Überlieferung von den drei Stadien dieses Entwicklungsganges hat, die charak-

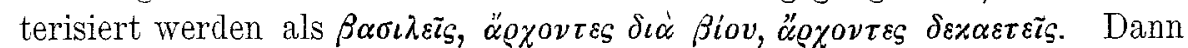
aber weiter, was wir denn eigentlich in den Trägern der vor Kreon in der Liste stehenden Namen zu sehen haben, ob Könige oder Archonten. 